\title{
¿Existen demasiadas sucursales bancarias en España? Un análisis para el periodo 1999-2011
}

\author{
David Conesa \\ Priscila Espinosa \\ Anabel Forte \\ Universitat de València \\ Emili Tortosa-Ausina \\ Universitat Jaume I
}

\section{Resumen}

Tras un largo periodo de intensa expansión territorial que culminó en 2008, la reestructuración del sector bancario español ha llevado a que el número de sucursales haya pasado de 45.000 a poco más de 28.000 en menos de una década. Las implicaciones de esta tendencia son notables, pues el acceso a la oficina física sigue siendo clave en comunidades remotas y/o desfavorecidas, además de existir un impacto todavía no medido en términos de banca relacional. El objetivo del trabajo consiste en determinar, a través de un modelo estadístico, si los fenómenos de over-branching y under-branching, esto es, exceso o escasez de oficinas, han estado presentes en el sector bancario español entre 1999 y 2011. La respuesta indicaría que la existencia de dichos fenómenos depende del tipo de entidad considerada (banco, caja o cooperativa), suponiendo la reestructuración del sector cajas de ahorros una amenaza para el acceso a los servicios bancarios en algunas regiones.

Palabras clave: banco, caja, cooperativa, municipio, sucursal.

Clasificación JEL: C11, G21, R1.

\begin{abstract}
After a long period of intense territorial expansion that peaked in 2008, the restructuring of the Spanish banking sector has led the number of bank branches to decline from 45,000 to just over 28,000 in less than a decade. The implications of this trend are remarkable, as access to the physical office remains key in remote and/or disadvantaged communities, and there is an impact not yet measured in terms of relationship banking. The objective of this paper is to determine, through a statistical model, whether the phenomena of over-branching and under-branching have been present in the Spanish banking sector between 1999 and 2011. The responses suggest that the existence of such phenomena depends on the type of bank considered (commercial bank, savings bank or credit union), with the restructuring of the savings banks sector threatening access to banking services in some regions.
\end{abstract}

Keywords: commercial bank, savings bank, county, bank branches.

JEL classification: C11, G21, R1. 


\section{Introducción}

Pese al cada vez mayor uso de la banca digital, no solo para la operativa más básica como la domiciliación de recibos o las transferencias sino incluso la contratación de depósitos bancarios o fondos de inversión, la importancia de la oficina bancaria física no puede ser soslayada. Como ha venido indicando Ergungor (2010), las entidades bancarias se especializan en prestar a clientes que, al menos a priori, son informacionalmente opacos y, por tanto, resulta clave el proceso de recopilación y procesamiento de información sobre los mismos. Como ha puesto de manifiesto repetidamente la literatura sobre banca relacional, obtener esta información puede resultar muy costoso, pero los costes pueden reducirse significativamente si existe una presencia física como es la oficina bancaria. Esta problemática afecta muy especialmente a áreas con niveles de renta relativamente bajos, en las que el acceso a la banca electrónica puede ser difícil (ya sea por tratarse de territorios remotos, por bajos niveles educativos de la población o por otras razones), que requeriría asesoramiento a la hora de contratar productos y servicios bancarios, contribuyendo así a una dependencia excesiva de canales no tradicionales en la relación con intermediarios bancarios (lo que podría llevar a mayores tipos de interés y comisiones más altas por los productos y servicios bancarios) y, en última instancia, a una persistencia en los niveles bajos de renta (Ergungor, 2010).

La importancia de la localización de oficinas bancarias adquiere mayor relevancia, si cabe, al repasar los campos en los que, de una u otra forma, se ha venido analizando el papel de la presencia física o la distancia a los intermediarios financieros. Entre estos, podemos mencionar en primer lugar la literatura que ha venido analizando el nexo entre finanzas y crecimiento económico (véanse King y Levine, 1993; Demirgüç-Kunt y Levine, 2001), una rama de la cual se ha ocupado de la disponibilidad de servicios financieros y acceso al crédito (Lanzillotti y Saving, 1969; Leyshon y Thrift, 1995, 1996; Pollard, 1996), así como la literatura sobre exclusión financiera (Carbó et al., 2007, 2005; Joassart-Marcelli y Stephens, 2010; Maudos, 2017), la banca relacional (Petersen y Rajan, 1995, 1994; Ongena y Smith, 2000, 2001; Elyasiani y Goldberg, 2004; Degryse y Ongena, 2005; Boot y Thakor, 2000; Boot, 2000), e incluso la literatura sobre micro créditos (Jain, 1996; Morduch, 1999). Estos son campos relevantes, en los que el número e importancia de muchas contribuciones son de tal calado, desde múltiples puntos de vista, que la hipótesis de que la distancia física ha dejado de ser relevante, al menos en lo que a productos y servicios bancarios se refiere, y pese a los grandes avances en la tecnología de la información y comunicaciones, debería ser descartada.

Por tanto, el análisis de temáticas relacionadas con la localización de oficinas bancarias y sus implicaciones, desde múltiples perspectivas, sigue siendo de una importancia capital. Sin embargo, pese al elevado número de trabajos, la gran mayoría de los mismos se centran en el caso de Estados Unidos (a excepción de la literatura sobre micro créditos), por lo que la problemática en otros contextos es menos conocida. Aunque existen estudios para otros países (por ejemplo, Okeahalam, 2009, 
para el caso de Sudáfrica), son relativamente escasos, y sus objetivos y resultados son muy diversos.

En el caso concreto que nos ocupa del sistema bancario español, si bien ha recibido considerablemente menos atención que el de Estados Unidos, tiene una importancia como actor fundamental entre los sistemas bancarios de la Unión Europea, así como por las elevadas cuotas de mercado de algunos bancos españoles en Latinoamérica (Santander y BBVA, fundamentalmente). Sin embargo, como veremos más adelante, existen otros motivos que han contribuido a la importancia relativa del caso español, entre los que deberíamos destacar en particular el proceso de reestructuración bancaria que tuvo lugar tras el pinchazo de la burbuja inmobiliaria y que afectó muy especialmente a las cajas de ahorros (Fernández-Villaverde et al., 2013).

Uno de los rasgos más destacables de este proceso de reestructuración bancaria ha sido el cierre, a fecha de diciembre de 2016, de un tercio de las oficinas bancarias que existían en 2008, cuando el número de sucursales llegó a su número más alto. Esta tendencia, por otro lado, parece además no haber llegado todavía a su fin. Este fenómeno excepcional (hasta el punto que podríamos referirnos al mismo como un experimento natural) tiene posiblemente su origen en el fuerte aumento del número de oficinas registrado entre 1992 y 2008, cuando prácticamente se duplicó. Estos fenómenos no han sido analizados con todo el detalle que requerirían, y ese será el objetivo del presente trabajo.

La importancia de esta problemática ha suscitado un interés notable, tanto desde un punto de vista de investigación como de política económica y, consecuentemente, numerosas contribuciones se han venido ocupando directamente del análisis de la localización de oficinas bancarias y sus implicaciones para el caso español. Por ejemplo, desde un punto de vista de economía industrial, algunos trabajos han analizado temáticas relacionadas con el contacto multi-mercado y similitud estratégica (Fuentelsaz y Gómez, 2006), decisiones de entrada y diversificación geográfica (Fuentelsaz y Gómez, 2001; Fuentelsaz et al., 2002), el modelo de submercados independientes (Sutton, 1997) y su aplicación al caso de la industria bancaria al por menor en España (De Juan, 2003), así como el examen de cómo las condiciones competitivas han cambiado en los mercados bancarios locales en España con anterioridad a la crisis (De Juan, 2008). Por contra, otros trabajos se han ocupado más directamente de los efectos que la desregulación bancaria puede haber tenido tanto sobre la productividad (Illueca et al., 2009), como sobre la propia viabilidad de las cajas de ahorros (Illueca et al., 2014), así como las implicaciones para el desarrollo y crecimiento regionales (Pastor et al., 2017).

Los estudios relacionados más directamente con el nuestro son aquellos que se han preocupado explícitamente de la localización geográfica de oficinas bancarias y de las implicaciones para la exclusión financiera. En concreto, tanto los trabajos de Bernad et al. (2008) como el de Alamá y Tortosa-Ausina (2012) han analizado cómo las decisiones de expansión geográfica pueden haber influido sobre el fenómeno de la exclusión financiera en España, uno de los posibles efectos colaterales derivados de la desregulación y reestructuración del sistema bancario español, debido al 
abandono de áreas más remotas y despobladas. Este estudio está relacionado con esos trabajos y, más concretamente, con el de Alamá et al. (2015), en el que el análisis de la localización de oficinas bancarias se evalúa a partir de un modelo estadístico, que permite definir los conceptos de under-branching y over-branching, esto es, falta o exceso de oficinas bancarias. Sin embargo, al centrarse este último trabajo únicamente en dos años (2004 y 2008), solo era posible evaluar parcialmente la dinámica de este convulso periodo.

En el presente trabajo, más concretamente, nos ocupamos de las tendencias observadas en la localización de oficinas bancarias en España durante un periodo relativamente dilatado, entre 1999 y 2011, que se inicia en unos años de expansión económica (una vez se cerró el proceso de consolidación bancaria de los años noventa), y que en su última parte corresponde a una situación de crisis. El final del periodo muestral se sitúa en 2011, dado que este es el último periodo para el que hay información disponible para todas las variables incluidas en el modelo. Por tanto, mientras Alamá et al. (2015) centraban su trabajo exclusivamente en los años 2004 y 2008, nuestro enfoque es más dinámico al cubrir también periodos anteriores (1999), centrales (2005) y posteriores (2009 y 2011) a dicho estudio, cubriendo un ciclo completo durante el cual se han producido tendencias contrapuestas, con un fuerte incremento en el número de oficinas hasta el máximo alcanzado en 2008 , y su posterior y rápido descenso.

El trabajo se estructura como sigue. Tras esta introducción, la segunda sección se ocupa de analizar desde un punto descriptivo algunas de las tendencias habidas en el sector bancario español en lo que a evolución del número de oficinas bancarias en España se refiere, así como su distribución territorial. En el tercer apartado se hace una breve descripción de los métodos utilizados en el estudio para, en el apartado 4, describir las variables utilizadas. Los resultados se presentan y comentan en la siguiente sección para, en último lugar, extraer algunas conclusiones derivadas del trabajo.

\section{Evolución del número de oficinas bancarias en España: 1988-2016}

Los fenómenos de expansión y contracción en la red de oficinas que han tenido lugar en España entre los años 1988 y 2016 han sido de tal intensidad que resulta difícil encontrar algún paralelismo en otros ámbitos geográficos. Entre las pocas excepciones existentes a nivel internacional se encuentra el caso de Estados Unidos donde en 1994, tras la aprobación de la Riegle-Neal Interstate Banking and Branching Efficiency Act, se permitió que los bancos se expandieran a otros Estados distintos al de su localización original. Sin embargo, el fenómeno fue de mucha menor intensidad comparado con el ocurrido en España, tanto por el menor impacto observado tras la desregulación estadounidense (en España se había realizado antes en 1988, a partir del Real Decreto-Ley 1582/1988, de 29 de diciembre), como por la ausencia de una reversión tan marcada del proceso como la ocurrida en España desde 2008. 
La intensidad del proceso se observa con claridad en el Cuadro 1.1, que proporciona información sobre la evolución del número de oficinas desde la aprobación y aplicación del Real Decreto-Ley 1582/1988 hasta los años más recientes. Podemos observar cómo, de acuerdo con lo apuntado en el párrafo anterior, desde 1988 el número de oficinas creció de una manera notable hasta los inicios de la crisis financiera (2008), pasando en esos años el número total de oficinas de 31.972 a 45.662 . Aunque el crecimiento en el conjunto de entidades bancarias fue muy elevado, este fenómeno fue especialmente intenso en el caso de las cajas de ahorro, entidades que se vieron notablemente beneficiadas por la desregulación y doblaron su número de oficinas en el mismo periodo (desde 12.252 en 1988 hasta 24.985 en 2008), llegando a representar casi el 55 por 100 del total de oficinas bancarias en España. Este tipo de entidades ha sido también el mayor protagonista del cierre de oficinas desde el inicio de la crisis, siendo este hecho también esperable por tratarse de las entidades más afectadas por la reestructuración del sector bancario, hasta el punto de haberse aprobado una nueva ley que las regula (Ley 26/2013, de 27 de diciembre, de cajas de ahorros y fundaciones bancarias).

Por lo que respecta a bancos y cooperativas, han sido menos partícipes de estas tendencias. En el primer caso, el número de oficinas llegó incluso a disminuir entre 1988 y 2008, de manera que mientras las cajas se expandían por la geografía española, en muchos casos fuera de sus mercados naturales (Fuentelsaz et al., 2004; Ilueca et al., 2009), en el caso de algunos bancos, especialmente los más grandes, se optó por una expansión internacional. Esta mayor diversificación internacional, y una menor implicación en el sector inmobiliario, ha propiciado que los bancos se hayan visto menos afectados por la crisis y, como consecuencia, la disminución en el número de oficinas haya sido mucho menor que en el caso de las cajas. Por su parte, la red de oficinas de las cooperativas también creció con claridad hasta 2008 para caer desde entonces, si bien a unas tasas más moderadas que en el caso de las cajas. Sin embargo, la futura ley de cooperativas de crédito, todavía en fases muy preliminares, podría incidir sobre estas tendencias.

Por su parte, el Cuadro 1.2 indica que existen notables diferencias en la evolución en el número de oficinas por provincia y comunidad autónoma, así como por tipo de entidad. Dichas diferencias son patentes tanto en la etapa de expansión (19882008) como en la reducción (2008-2016) del número de oficinas. Como indican los estadísticos descriptivos del final del cuadro, el crecimiento anual promedio en el periodo de expansión fue del 1,07 por 100, pero la desviación típica fue notable $(0,99)$, subyaciendo una distribución geográfica dispar en la expansión territorial. Así, mientras la tendencia general fue la de aumento en el número de oficinas, en algunas provincias, caracterizadas por un menor dinamismo económico, hubo una caída. Es el caso de Huesca, Ciudad Real, Cuenca, Palencia, Soria, Cáceres, Lugo y Ourense. Por tanto, a falta de un análisis más detallado, podríamos concluir que la dificultad en el acceso a los servicios financieros y bancarios a través de la oficina física no era un fenómeno desconocido con anterioridad al estallido de la crisis. En el otro extremo se encontrarían los casos de provincias como Almería, Las Palmas, 


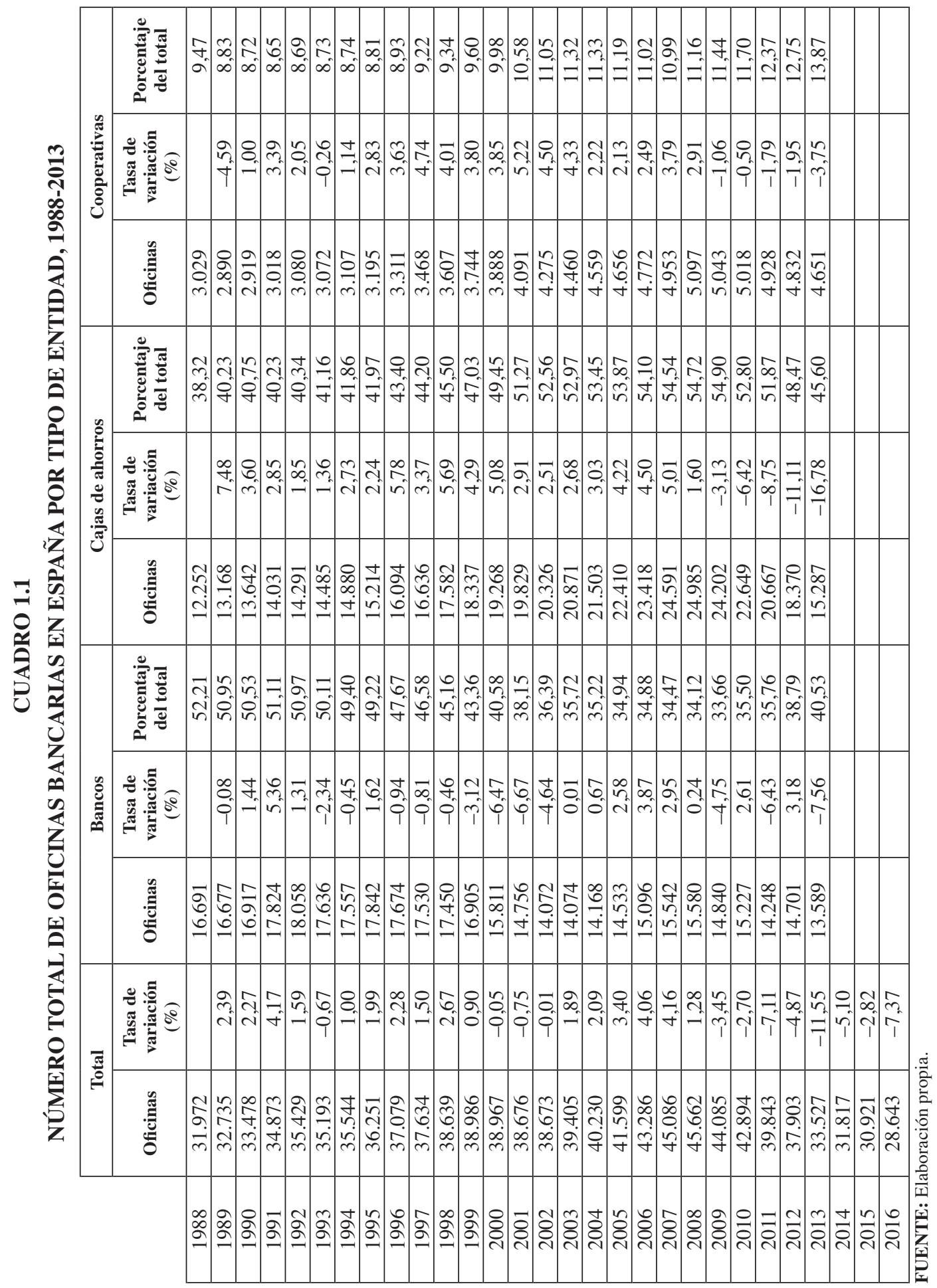


Madrid y Murcia, con variaciones anuales promedio superiores al 3 por 100 durante el mismo periodo.

Tras el estallido de la crisis y después de la reestructuración del sector bancario (2008-2016), y como se ha venido apuntando en los párrafos anteriores, la tendencia ha sido opuesta, habiéndose alcanzado valores de la densidad en la red de oficinas similares a los existentes en los años ochenta del siglo pasado. Sin embargo, tras esta tendencia general, que no tiene paralelismo en ningún otro país, encontramos mayores disparidades que las existentes en el periodo 1988-2008. Como se observa en las últimas filas del Cuadro 1.2, en este caso el promedio es de mucha mayor magnitud $(-4,97$ por 100$)$, y una desviación típica más elevada ( 1,53 frente al 0,99 correspondiente al periodo anterior) nos estaría indicando que el fenómeno del cierre de oficinas está siendo intenso pero también que no en todas las provincias ha sido del mismo calado. En este caso, se observa que en varias provincias el proceso de cierre de oficinas ha sido más intenso que en la media nacional, con tasas negativas por debajo de -7 por 100 . Son los casos de Zaragoza, Barcelona, Girona, Tarragona, Castelló, Ourense, mientras que en el otro extremo encontraríamos las provincias de Teruel, Ciudad Real, Cuenca, Badajoz, Guipúzcoa, con caídas en el entorno del -2 por 100. Por tanto, a falta de un análisis más pormenorizado (como, por ejemplo, el llevado a cabo en Alamá y Tortosa-Ausina (2012) y Maudos (2017), los resultados podrían estar apuntando a que, por una parte, una mayor integración bancaria puede contribuir y a la vez frenar el crecimiento económico, como se indica en los trabajos de Loutskina y Strahan (2015) y Gilje et al. (2016) y, por otra, que los procesos de reestructuración bancaria pueden haber llevado a que en algunas provincias como Ourense el número de oficinas haya caído en los dos periodos, con el consiguiente deterioro en el acceso a los servicios financieros y bancarios ${ }^{1}$.

De algunos de estos hechos dan cuenta los Gráficos 1.1 y 1.2, en los que se representan las tasas de crecimiento promedio para cada comunidad autónoma. Como se puede comprobar, las regiones en las que las redes de oficinas crecieron más (menos) no han sido necesariamente aquellas en las que, a partir de 2008, la caída ha sido más intensa. Por ejemplo, las comunidades líderes en el cierre de oficinas están siendo Cataluña y la Comunidad Valenciana (Gráfico 1.2), pero no lo fueron en el periodo de expansión (Gráfico 1.1). Por tanto, los motivos de la expansión y contracción en la red de oficinas podrían no ser necesariamente los mismos, pudiendo ser un factor explicativo la reestructuración del sistema bancario español. Por otra parte, este proceso de cambios en el sistema bancario podría haber contribuido a ralentizar la recuperación económica de algunas regiones.

\footnotetext{
${ }^{1}$ Esta literatura reciente postula que, cuando las redes de oficinas son más densas, la integración bancaria es mayor porque regiones relativamente remotas y con lo que bajo circunstancias normales constituiría un difícil acceso a productos y servicios bancarios, se ve facilitado por la mayor presencia de oficinas bancarias.
} 


\section{CUADRO 1.2}

\section{DISTRIBUCIÓN DE OFICINAS BANCARIAS Y SU VARIACIÓN Y POR PROVINCIAS, 1988-2016}

\begin{tabular}{|c|c|c|c|c|c|c|c|}
\hline $\begin{array}{l}\text { Provincia/ } \\
\text { Comunidad } \\
\text { Autónoma }\end{array}$ & 1988 & 2008 & $\begin{array}{c}\text { Tasa de va- } \\
\text { riación anual } \\
\text { promedio, } \\
1988-2008\end{array}$ & 2016 & $\begin{array}{c}\text { Tasa de va- } \\
\text { riación anual } \\
\text { promedio, } \\
2008-2016\end{array}$ & $\begin{array}{c}\text { Tasa de va- } \\
\text { riación anual } \\
\text { promedio, } \\
\text { 1988-2016 }\end{array}$ & $\begin{array}{c}\text { Suma de las } \\
\text { tasas de varia- } \\
\begin{array}{c}\text { ción en valor } \\
\text { absoluto }\end{array} \\
\end{array}$ \\
\hline Almería & 382 & 705 & 3,11 & 457 & $-5,27$ & 0,64 & \\
\hline Cádiz & 550 & 793 & 1,85 & 493 & $-5,77$ & $-0,39$ & \\
\hline Córdoba & 565 & 687 & 0,98 & 498 & $-3,94$ & $-0,45$ & \\
\hline Granada & 565 & 844 & 2,03 & 596 & $-4,26$ & 0,19 & \\
\hline Huelva & 371 & 444 & 0,90 & 287 & $-5,31$ & $-0,91$ & \\
\hline Jaén & 497 & 630 & 1,19 & 484 & $-3,24$ & $-0,09$ & \\
\hline Málaga & 790 & 1393 & 2,88 & 824 & $-6,35$ & 0,15 & \\
\hline Sevilla & 950 & 1514 & 2,36 & 933 & $-5,87$ & $-0,06$ & \\
\hline Andalucía & 4.670 & 7.010 & 2,05 & 4572 & $-5,20$ & $-0,08$ & 7,25 \\
\hline Huesca & 392 & 358 & $-0,45$ & 241 & $-4,83$ & $-1,72$ & \\
\hline Teruel & 215 & 236 & 0,47 & 189 & $-2,74$ & $-0,46$ & \\
\hline Zaragoza & 897 & 1202 & 1,47 & 665 & $-7,13$ & $-1,06$ & \\
\hline Aragón & 1.504 & 1.796 & 0,89 & 1.095 & $-6,00$ & $-1,13$ & 6,89 \\
\hline Asturias, & 816 & 966 & 0,85 & 708 & $-3,81$ & $-0,51$ & 4,66 \\
\hline Baleares & 858 & 1.254 & 1,92 & 852 & $-4,72$ & $-0,03$ & 6,63 \\
\hline Las Palmas & 411 & 761 & 3,13 & 460 & $-6,10$ & 0,40 & \\
\hline Sta. Cruz de Tenerife & 471 & 702 & 2,02 & 486 & $-4,49$ & 0,11 & \\
\hline Canarias & 882 & 1.463 & 2,56 & 946 & $-5,30$ & 0,25 & $\mathbf{7 , 8 7}$ \\
\hline Cantabria & 487 & 503 & 0,16 & 362 & $-4,03$ & $-1,05$ & 4,19 \\
\hline Albacete & 290 & 348 & 0,92 & 263 & $-3,44$ & $-0,35$ & \\
\hline Ciudad Real & 506 & 474 & $-0,33$ & 398 & $-2,16$ & $-0,85$ & \\
\hline Cuenca & 258 & 254 & $-0,08$ & 216 & $-2,01$ & $-0,63$ & \\
\hline Guadalajara & 201 & 273 & 1,54 & 197 & $-4,00$ & $-0,07$ & \\
\hline Toledo & 588 & 669 & 0,65 & 524 & $-3,01$ & $-0,41$ & \\
\hline Castilla-La Mancha & 1.843 & 2.018 & 0,45 & 1598 & $-2,87$ & $-0,51$ & 3,33 \\
\hline Ávila & 187 & 217 & 0,75 & 135 & $-5,76$ & $-1,16$ & \\
\hline Burgos & 463 & 547 & 0,84 & 360 & $-5,09$ & $-0,89$ & \\
\hline León & 430 & 547 & 1,21 & 401 & $-3,81$ & $-0,25$ & \\
\hline Palencia & 254 & 224 & $-0,63$ & 145 & $-5,29$ & $-1,98$ & \\
\hline Salamanca & 365 & 407 & 0,55 & 267 & $-5,13$ & $-1,11$ & \\
\hline Segovia & 179 & 208 & 0,75 & 136 & $-5,17$ & $-0,98$ & \\
\hline Soria & 172 & 150 & $-0,68$ & 108 & $-4,02$ & $-1,65$ & \\
\hline Valladolid & 501 & 606 & 0,96 & 369 & $-6,01$ & $-1,09$ & \\
\hline Zamora & 242 & 256 & 0,28 & 186 & $-3,91$ & $-0,94$ & \\
\hline Castilla-León & 2.793 & 3.162 & 0,62 & 2107 & $-4,95$ & $-1,00$ & 5,57 \\
\hline
\end{tabular}

FUENTE: Elaborción propia. 


\section{CUADRO 1.2 (continuación) \\ DISTRIBUCIÓN DE OFICINAS BANCARIAS Y SU VARIACIÓN Y POR PROVINCIAS, 1988-2016}

\begin{tabular}{|c|c|c|c|c|c|c|c|}
\hline $\begin{array}{l}\text { Provincia/ } \\
\text { Comunidad } \\
\text { Autónoma }\end{array}$ & 1988 & 2008 & $\begin{array}{c}\text { Tasa de va- } \\
\text { riación anual } \\
\text { promedio, } \\
\text { 1988-2008 }\end{array}$ & 2016 & $\begin{array}{c}\text { Tasa de va- } \\
\text { riación anual } \\
\text { promedio, } \\
2008-2016\end{array}$ & $\begin{array}{c}\text { Tasa de va- } \\
\text { riación anual } \\
\text { promedio, } \\
1988-2016\end{array}$ & $\begin{array}{c}\text { Suma de } \\
\text { las tasas de } \\
\text { variación en } \\
\text { valor absoluto }\end{array}$ \\
\hline Barcelona & 4.598 & 5.819 & 1,18 & 2.916 & $-8,27$ & $-1,61$ & \\
\hline Girona & 737 & 858 & 0,76 & 458 & $-7,55$ & $-1,68$ & \\
\hline Lleida & 585 & 588 & 0,03 & 368 & $-5,69$ & $-1,64$ & \\
\hline Tarragona & 711 & 833 & 0,79 & 447 & $-7,49$ & $-1,64$ & \\
\hline Catalunya & 6.631 & 8.098 & 1,00 & 4.180 & $-7,91$ & $-1,63$ & 8,91 \\
\hline Alicante/Alacant & 1.247 & 1.736 & 1,67 & 1.046 & $-6,14$ & $-0,63$ & \\
\hline Castellón/Castelló & 507 & 679 & 1,47 & 371 & $-7,28$ & $-1,11$ & \\
\hline Valencia/València & 1.794 & 2.646 & 1,96 & 1.515 & $-6,73$ & $-0,60$ & \\
\hline Com. Valenciana & 3.548 & 5.061 & 1,79 & 2.932 & $-6,60$ & $-0,68$ & 8,39 \\
\hline Badajoz & 489 & 747 & 2,14 & 619 & $-2,32$ & 0,85 & \\
\hline Cáceres & 460 & 450 & $-0,11$ & 345 & $-3,27$ & $-1,02$ & \\
\hline Extremadura & 949 & 1.197 & 1,17 & 964 & $-2,67$ & 0,06 & 3,84 \\
\hline Coruña, A & 785 & 982 & 1,13 & 642 & $-5,17$ & $-0,72$ & \\
\hline Lugo & 357 & 336 & $-0,30$ & 248 & $-3,72$ & $-1,29$ & \\
\hline Ourense & 430 & 374 & $-0,70$ & 204 & $-7,30$ & $-2,63$ & \\
\hline Pontevedra & 710 & 820 & 0,72 & 497 & $-6,07$ & $-1,27$ & \\
\hline Galicia & 2.282 & 2.512 & 0,48 & 1.591 & $-5,55$ & $-1,28$ & 6,03 \\
\hline Madrid & 2.958 & 6.023 & 3,62 & 3.553 & $-6,38$ & 0,66 & 10,00 \\
\hline Murcia & 739 & 1.353 & 3,07 & 852 & $-5,62$ & $\mathbf{0 , 5 1}$ & 8,69 \\
\hline Navarra & 657 & 716 & $\mathbf{0 , 4 3}$ & 531 & $-3,67$ & $-0,76$ & 4,10 \\
\hline Álava/Araba & 295 & 348 & 0,83 & 219 & $-5,62$ & $-1,06$ & \\
\hline Guipúzcoa/Gipuzkoa & 563 & 594 & 0,27 & 486 & $-2,48$ & $-0,52$ & \\
\hline Vizcay/Bizkaia & 801 & 1.046 & 1,34 & 721 & $-4,54$ & $-0,38$ & \\
\hline País Vasco & 1.659 & 1.988 & $\mathbf{0 , 9 1}$ & 1.426 & $-4,07$ & $-0,54$ & 4,98 \\
\hline Rioja, La & 447 & 496 & 0,52 & 327 & $-5,07$ & $-1,11$ & 5,60 \\
\hline Media & & 1,07 & & $-4,97$ & $-0,70$ & & \\
\hline Desviación típica & & 0,99 & & 1,53 & 0,71 & & \\
\hline Mediana & & 0,91 & & $-5,15$ & $-0,70$ & & \\
\hline Máximo & & 3,62 & & $-2,01$ & 0,85 & & \\
\hline Mínimo & & $-0,70$ & & $-8,27$ & $-2,63$ & & \\
\hline
\end{tabular}

FUENTE: Elaborción propia. 


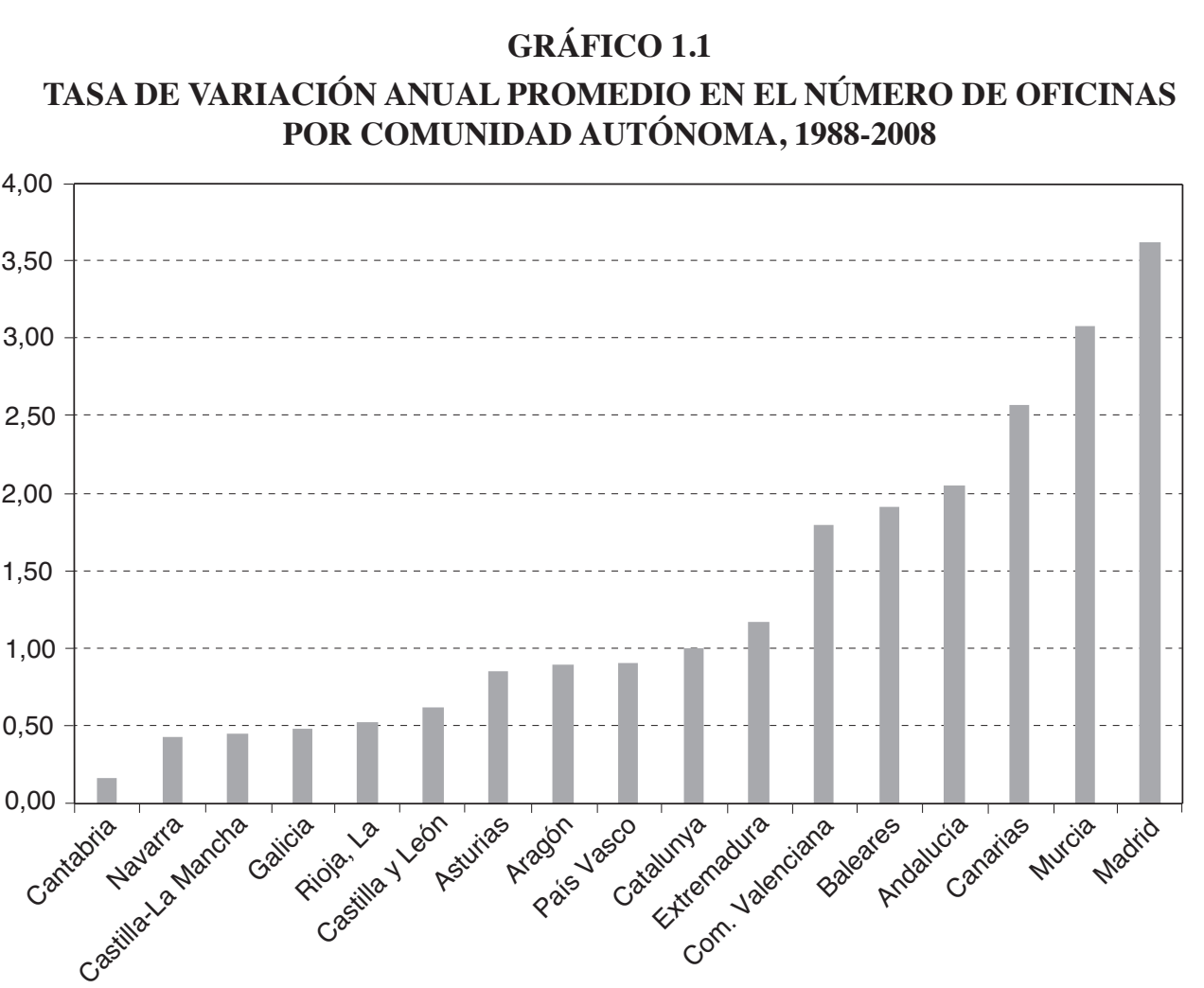

FUENTE: Elaborción propia.

\section{Metodología}

En este trabajo, para poder valorar los determinantes de la localización de las oficinas bancarias en España, se ha utilizado un modelo que permite describir la distribución del número de oficinas observadas en cada uno de los 7.177 municipios españoles de la muestra. En concreto, se trata de un modelo de regresión de Poisson mixto -véase, por ejemplo, McCulloch y Searle (2011) para una descripción detallada de este tipo de modelos- que relaciona el número medio de oficinas en una determinada localización con las características de la misma. En esta sección describimos dicha modelización así como el proceso inferencial sobre los parámetros de interés.

De manera similar a Alamá et al. (2015), para cada uno de los años analizados, el número de oficinas bancarias para un municipio $i$ es denotado por $O_{i}$ y puede ser modelizado utilizando una distribución de Poisson con media $\mu_{i}$

Esta media representa el número de oficinas bancarias esperadas en un municipio e identifica totalmente el comportamiento de la variable. Para su modelización en este trabajo nos hemos focalizado en tres aspectos que consideramos de especial relevancia. Por un lado, consideramos que el número esperado de oficinas vendrá 


\section{GRÁFICO 1.2}

\section{TASA DE VARIACIÓN ANUAL PROMEDIO EN EL NÚMERO DE OFICINAS POR COMUNIDAD AUTÓNOMA, 2008-2016}

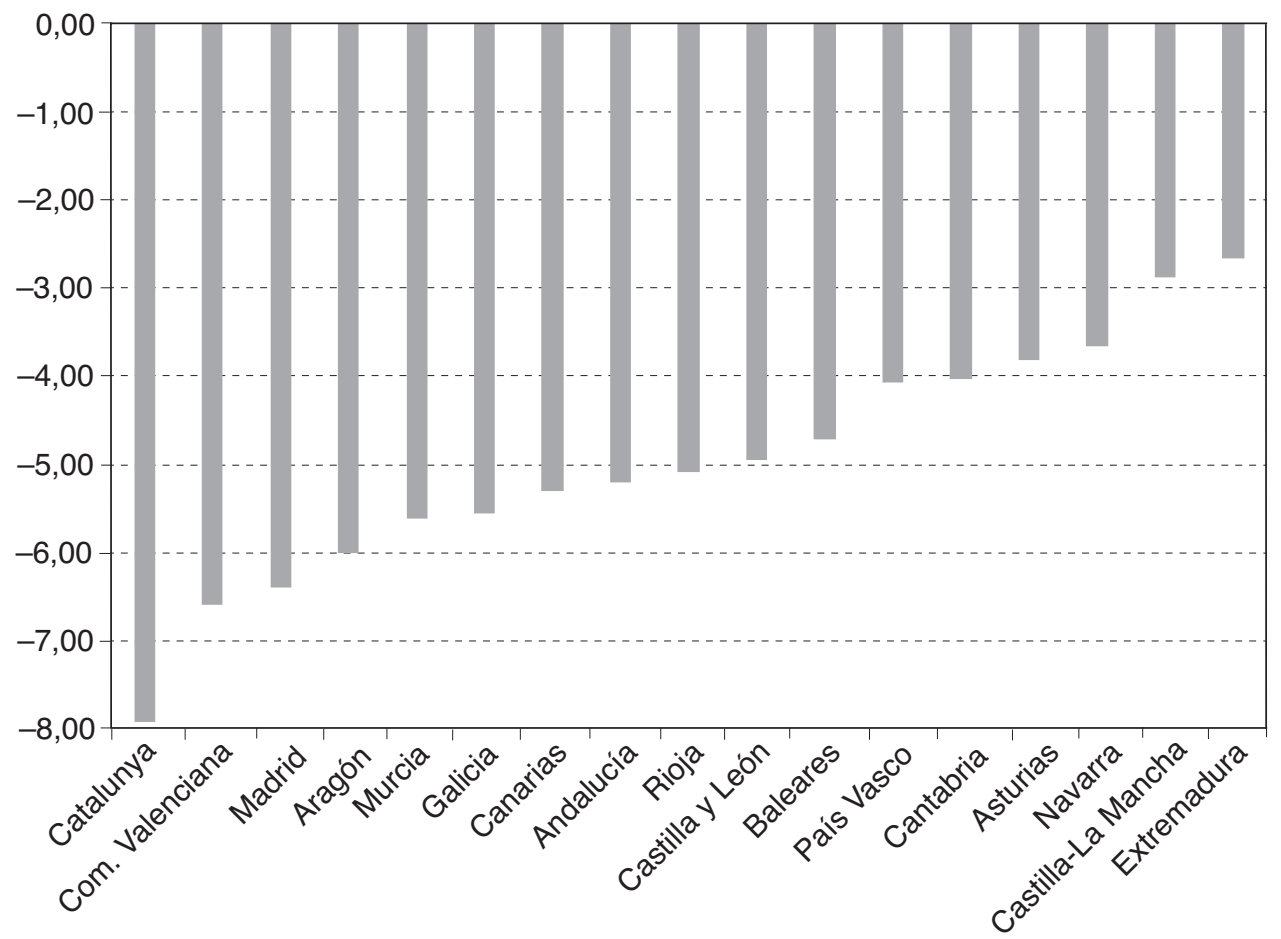

FUENTE: Elaborción propia.

determinado por la población que pueda tener acceso a las mismas, esto es, el tamaño del municipio. Además, este número se verá condicionado por algunas características socioeconómicas específicas de cada municipio, que expresaremos aquí en forma de covariables. Por último, cabe esperar un efecto propio de los municipios y/o provincias que propicie observar más o menos sucursales de las esperadas por población.

Estas tres fuentes de variabilidad se pueden incorporar en el modelo anterior mediante la descomposición del número esperado de sucursales, en:

- El número de entidades esperadas en relación a la cantidad de personas con acceso a las mismas, o dicho de otra forma, la proporción de oficinas por individuo multiplicada por la población de cada municipio.

- El efecto de las condiciones socioeconómicas representadas por las covariables consideradas. Se tratará de un efecto multiplicativo que modificará el número esperado de oficinas por individuo. 
- Los efectos de factores no observables a nivel tanto municipal como provincial, y que se introducen en el modelo a través de efectos aleatorios.

De estos tres términos, los dos primeros nos permiten establecer el número de oficinas esperadas en un municipio con la información disponible y el tercero es el que aporta una medida del posible exceso (over-branching si $>0$ ) o falta (under-branching si $<0)$ de oficinas en ese municipio concreto. Hasta el momento, este tipo de medidas solo han sido anteriormente planteadas y utilizadas en el trabajo de Alamá et al. (2015).

La estimación de los parámetros del modelo se ha realizado desde una perspectiva bayesiana objetiva, utilizado la aproximación INLA (Integrated Nested Laplace Aproximation) propuesta por Rue et al. (2009).

\section{Datos y variables}

En este trabajo se utilizan diferentes conjuntos de datos proporcionados por distintas instituciones. En cuanto a los datos sobre la ubicación geográfica de las sucursales bancarias, estos han sido obtenidos a partir de una Guía de la Banca, que proporciona información sobre la dirección postal completa de cada sucursal bancaria en España. Los años seleccionados para la muestra son 1999, 2005, 2009 y 2011.

En el año inicial del periodo muestral (1999), ya se había producido la primera gran ola de fusiones en el sector bancario español, que afectó principalmente a las cajas de ahorros, y se había iniciado un periodo en el que muchas de ellas habían emprendido políticas de expansión territorial ambiciosas. Los años 2005 y 2009 son también relevantes por tratarse de periodos en los que la economía estaba, respectivamente, en plena expansión y desaceleración. El periodo analizado finaliza, forzosamente, en 2011 por tratarse del último año para el que hay información disponible para todas las variables incluidas en el modelo. Aunque, idealmente, hubiera sido aún más interesante disponer de la información lo más actualizada posible, y de hecho se dispone de información relativa a la localización geográfica de oficinas hasta 2015, las dificultades para actualizar otras variables limitan el alcance temporal del estudio. Dado que el proceso de racionalización de la red de oficinas bancarias todavía no se ha cerrado, una de las extensiones del estudio será su actualización en un periodo razonable.

Por otra parte, el estudio utiliza otro conjunto de variables relacionadas con los factores que pueden determinar el número de sucursales bancarias en un lugar determinado, como se documenta en las secciones anteriores. En el caso particular de la banca española, los estudios de Alamá y Tortosa-Ausina (2012) y Bernad et al. (2008) han considerado un número relativamente grande de variables socioeconómicas. Sin embargo, estos estudios tienen en parte un sesgo geográfico en el sentido de que incluyen solo los municipios con más de 1000 habitantes, que representan menos del 70 por 100 del territorio español. Por tanto, teniendo en cuenta las crecientes 
dificultades a las que se enfrentan amplios segmentos de población (especialmente aquellos que viven en zonas rurales relativamente remotas o núcleos urbanos que han perdido su sucursal), consideramos relevante extender la muestra a estos municipios, ganando así precisión desde un punto de vista de acceso físico a servicios bancarios y exclusión financiera. Aunque este enfoque supone dejar de lado algunas de las variables que se consideran en los estudios de Alamá y Tortosa-Ausina (2012) y Bernad et al. (2008), el número de municipios incluidos en el estudio aumenta sustancialmente, abarcando una muestra representativa de ellos (7.177 de un total de 8.109 posibles) y, por tanto, de todo el territorio español ${ }^{2}$.

El conjunto de covariables contiene algunos de los determinantes más frecuentes considerados en la literatura sobre acceso físico a servicios bancarios. Entre ellas, es habitual la inclusión de la renta per cápita, si bien esta información no está disponible para todos los municipios y años de la muestra. Alternativamente, usamos el desempleo, que está fuertemente correlacionado (negativamente) con los niveles de renta, a partir de la información proporcionada por el Ministerio de Empleo y Seguridad Social.

También se considera como una variable relevante la densidad de población, también considerada en otros estudios como, por ejemplo, por Evanoff (1988) y, anteriormente, Lanzillotti y Saving (1969). Como indican Bernad et al. (2008), sería razonable esperar que un aumento en la densidad de población reduzca el número de sucursales bancarias a través del efecto de unos menores costes para atender a la demanda de servicios bancarios. Cabe desatacar, sin embargo, que, en nuestro caso particular, podríamos obtener conclusiones distintas fruto de las diferencias entre nuestros datos y los de Bernad et al. (2008), ya que muchos de los municipios incluidos en nuestra muestra tienen una densidad de población muy baja.

La tercera de las covariables seleccionadas corresponde a la población extranjera, calculada como el porcentaje de personas de nacionalidad no española sobre la población total del municipio. El número de autores que han utilizado este tipo de información en sus estudios es relativamente bajo debido a su falta de disponibilidad para muchos países. Una de las pocas excepciones es el trabajo de Joassart-Marcelli y Stephens (2010), cuyo análisis, basado en la literatura sobre la exclusión financiera y ecología, se ocupa de las relaciones espaciales entre los patrones de asentamiento de inmigrantes en el área metropolitana de Boston en el año 2000, así como del análisis de la accesibilidad a diversos tipos de servicios proporcionados por instituciones financieras. Cabe destacar que, el efecto de esta variable se verá condicionado por la situación económica de los inmigrantes y, por tanto, es factible encontrar resultados distintos para estudios realizados en diferentes países.

Por supuesto, existen otras covariables que pueden tener un efecto sobre el número de sucusales. Por ejemplo, en la literatura se habla de la relación existente entre la localización de las sucursales bancarias y la actividad económica en general que puede descomponerse en diferentes variables como el turismo, las actividades

${ }^{2}$ En la muestra final algunos municipios fueron eliminados por la falta de datos para dicha localidad. 
comerciales (tanto al por menor como al por mayor), el número de camiones, etc., pero no se ha podido disponer de datos para ser incluidas ${ }^{3}$.

El último elemento en la modelización del número medio de sucursales bancarias es el efecto de la provincia introducido como un término de variabilidad que incorpora a la media aquellos efectos propios de una provincia y que no se engloban dentro de los efectos propios de las covariables. Aunque con anterioridad al cambio legislativo de 1988 (Real Decreto-Ley 1582/1988, de 29 de diciembre) las cajas de ahorros ya estaban autorizadas a expandirse geográficamente dentro de sus regiones de origen (básicamente comunidades autónomas), esto solo se llevó a cabo a tasas moderadas, si bien dependía también de la caja de ahorros en cuestión (véase Fuentelsaz et al., 2004). Además, algunas regulaciones existentes con anterioridad les habían impedido expandirse fuera de sus provincias. Por tanto, parece justificada la inclusión un efecto geográfico restringido a la provincia. Desde un punto de vista estadístico, modelizamos este efecto provincial a través de efectos aleatorios independientes ${ }^{4}$.

Por último, y para dar cabida a algunas teorías tradicionales de gran calado en el campo de la economía industrial como son la teoría del rational herding o «seguimiento racional» (Chang et al., 1997), y la de rival precedence, o «decisiones previas tomadas por rivales» (Hannan y McDowell, 1987) ${ }^{5}$, se estudiarán por separado el número de entidades bancarias de cada tipo (bancos comerciales, cajas de ahorro y cooperativas de crédito) introduciendo, como covariables, el número de sucursales de otros tipos existentes en cada uno de los municipios.

\section{Resultados}

La modelización estadística considerada se aplicará en cuatro escenarios diferentes. En primer lugar, podemos considerar que no existen diferencias entre tipos de bancos y, por tanto, la variable respuesta sería el número total de oficinas bancarias en cada municipio. Después se pasará a considerar como variable respuesta el número de oficinas correspondientes a bancos comerciales, cajas de ahorros y cooperativas de crédito por separado. Este tipo de estrategia viene motivada por las distintas implicaciones para la exclusión financiera (que se correspondería con el caso de falta de oficinas o under-branching) de los diferentes tipos de entidades, o el hecho de que

Dicha información, para el caso español, era proporcionada por el Anuario Económico de España de La Caixa, si bien únicamente para municipios de más de 1.000 habitantes, lo que imposibilita su utilización en nuestro trabajo.

${ }^{4}$ En el estudio se consideró también la posibilidad de incluir otra fuente de variabilidad como es el efecto del municipio. Sin embargo, las estimaciones iniciales mostraron que la ganancia en complejidad del modelo y dificultad de cálculo no compensaban la variabilidad adicional explicada, siendo por tanto poco razonable la inclusión de esta componente municipal.

${ }^{5}$ El concepto de precedencia rival ha sido utilizado con intensidad por la literatura sobre economía industrial y hace referencia a cómo las empresas reaccionan ante el comportamiento observado de los rivales al tomar decisiones relevantes tales como la adopción de una nueva tecnología. 
las cajas de ahorros serían el agregado institucional que, hasta el momento, ha estado sometido a la más profunda reestructuración y que más se expandió territorialmente durante los años de mayor crecimiento económico.

Los resultados relativos al efecto de las variables analizadas sobre el número total de oficinas bancarias, independientemente del tipo de entidad, aparecen en los Cuadros 2.1, 2.2, 2.3 y 2.4 para el conjunto de entidades, bancos comerciales, cajas de ahorros y cooperativas de crédito, respectivamente. Cada uno de los cuadros se divide en cuatro paneles, correspondientes a los años objeto de estudio $(1999,2005$, 2009 y 2011).

Como podemos observar en el Cuadro 2.1, para el conjunto de las entidades el impacto de la densidad de población sobre el número esperado de oficinas bancarias es negativo, tanto si tenemos en cuenta el efecto promedio como analizando los distintos cuantiles, observándose una ligera tendencia decreciente (en valor absoluto) desde 2005. Por tanto, se tendría que, cuanto más dispersa está la población, la proporción de oficinas por individuo aumenta. Si bien este resultado podría parecer, a priori, contrario a lo que cabría esperar (esto es, más oficinas bancarias en zonas más pobladas), una explicación cuidadosa evitará interpretaciones equivocadas. Así, el efecto negativo se debería al hecho de que el número de oficinas estaría por debajo del esperado para el tamaño poblacional del municipio, teniendo en cuenta el resto de efectos recogidos por el modelo. Al descomponer el efecto por tipo de entidad (Cuadros 2.2, 2.3 y 2.4 para bancos, cajas y cooperativas, respectivamente), el signo se mantiene pero la magnitud del mismo aumenta siendo mucho mayor para los dos agregados institucionales con un mayor arraigo en sus regiones de origen (cajas y cooperativas), y resulta especialmente elevado (en valor absoluto) en el caso de las cooperativas. Por tanto, se confirmaría el mayor compromiso de estas instituciones financieras con zonas rurales así como comunidades que residen en zonas más remotas y/o despobladas. En cualquier caso, independientemente del tipo de entidad, la magnitud del efecto (esto es, el coeficiente en valor absoluto) ha caído claramente en los últimos años, especialmente en el caso de las cooperativas, cuyo efecto promedio ha pasado de -0,3873 en 1999 a -0,3161 en 2011 (véase Cuadro 2.4) indicando una disminución del efecto de la densidad poblacional a medida que avanzan los años. Este efecto puede observarse también en las filas correspondientes a las IRR. Las IRR son las Incidence Rate Ratio o «tasa de incidencia relativa» que nos marcan el aumento, en términos multiplicativos, del numero esperado de oficinas al aumentar una unidad la variable de interés. Esto es, un IRR de 1 nos indicaría que el número esperado de oficinas queda igual sin importar el valor de esa variable mientras que un IRR $>1(<1)$ nos indicaría un aumento (descenso) en el número esperado al aumentar el valor de la variable. Teniendo esto en mente, podemos observar que el IRR asociado a la densidad de población se va acercando a 1 con los años indicando, como exponíamos anteriormente, una disminución en el efecto de esta variable.

Asimismo, la información relativa a la ratio entre número de oficinas observadas y esperadas se muestra en las filas correspondientes a la tasa de incidencia relativa (IRR), y en ellas se observa de nuevo el mayor compromiso de las cooperativas, al 
CUADRO 2.1

DISTRIBUCIONES A POSTERIORI DE LOS COEFICIENTES DE REGRESIÓN, TOTAL (1999, 2005, 2009 Y 2011)

\begin{tabular}{|c|c|c|c|c|c|c|c|}
\hline \multicolumn{8}{|c|}{1999} \\
\hline & Media & s.d. & $\begin{array}{c}2,5 \% \\
\text { cuantil }\end{array}$ & $\begin{array}{c}25 \% \\
\text { cuantil }\end{array}$ & $\begin{array}{c}50 \% \\
\text { cuantil }\end{array}$ & $\begin{array}{c}75 \% \\
\text { cuantil }\end{array}$ & $\begin{array}{l}97,5 \% \\
\text { cuantil }\end{array}$ \\
\hline Intercept & 1,1934 & 0,0401 & 1,1141 & 1,1666 & 1,1935 & 1,2203 & 1,2719 \\
\hline Densidad población & $-0,1689$ & 0,0034 & $-0,1755$ & $-0,1712$ & $-0,1689$ & $-0,1667$ & $-0,1623$ \\
\hline Densidad IRR & 0,8446 & 0,8390 & 0,8427 & 0,8446 & 0,8465 & 0,8502 & 0,8446 \\
\hline Tasa desempleo & $-0,0459$ & 0,0032 & $-0,0522$ & $-0,0481$ & $-0,0459$ & $-0,0437$ & $-0,0395$ \\
\hline Tasa desempleo IRR & 0,9552 & 0,9491 & 0,9531 & 0,9552 & 0,9573 & 0,9613 & 0,9552 \\
\hline $\begin{array}{l}\text { Porcentaje población } \\
\text { extranjera }\end{array}$ & 0,0040 & 0,0015 & 0,0010 & 0,0030 & 0,0041 & 0,0051 & 0,0070 \\
\hline $\begin{array}{l}\text { Porcentaje población } \\
\text { extranjera IRR }\end{array}$ & 1,0041 & 1,0010 & 1,0030 & 1,0041 & 1,0051 & 1,0071 & 1,0041 \\
\hline s.d.-Provincias & 0,2412 & 0,0256 & 0,1965 & 0,2229 & 0,2391 & 0,2571 & 0,2970 \\
\hline \multicolumn{8}{|c|}{2005} \\
\hline & Media & s.d. & $\begin{array}{c}2,5 \% \\
\text { cuantil }\end{array}$ & $\begin{array}{c}25 \% \\
\text { cuantil }\end{array}$ & $\begin{array}{c}50 \% \\
\text { cuantil }\end{array}$ & $\begin{array}{c}75 \% \\
\text { cuantil }\end{array}$ & $\begin{array}{l}97,5 \% \\
\text { cuantil }\end{array}$ \\
\hline Intercept & 1,0044 & 0,0411 & 0,9234 & 0,9769 & 1,0044 & 1,0319 & 1,0852 \\
\hline Densidad población & $-0,1885$ & 0,0031 & $-0,1946$ & $-0,1906$ & $-0,1886$ & $-0,1865$ & $-0,1825$ \\
\hline Densidad IRR & 0,8282 & 0,8232 & 0,8264 & 0,8282 & 0,8299 & 0,8332 & 0,8282 \\
\hline Tasa desempleo & 0,0004 & 0,0013 & $-0,0023$ & $-0,0004$ & 0,0005 & 0,0013 & 0,0027 \\
\hline Tasa desempleo IRR & 1,0004 & 0,9977 & 0,9996 & 1,0005 & 1,0013 & 1,0028 & 1,0004 \\
\hline $\begin{array}{l}\text { Porcentaje población } \\
\text { extranjera }\end{array}$ & 0,0031 & 0,0009 & 0,0014 & 0,0025 & 0,0031 & 0,0037 & 0,0049 \\
\hline $\begin{array}{l}\text { Porcentaje población } \\
\text { extranjera IRR }\end{array}$ & 1,0031 & 1,0014 & 1,0025 & 1,0031 & 1,0037 & 1,0049 & 1,0031 \\
\hline s.d.-Provincias & 0,2606 & 0,0271 & 0,2131 & 0,2412 & 0,2584 & 0,2775 & 0,3198 \\
\hline \multicolumn{8}{|c|}{2009} \\
\hline & Media & s.d. & $\begin{array}{c}2.5 \% \\
\text { cuantil }\end{array}$ & $\begin{array}{c}25 \% \\
\text { cuantil }\end{array}$ & $\begin{array}{c}50 \% \\
\text { cuantil }\end{array}$ & $\begin{array}{c}75 \% \\
\text { cuantil }\end{array}$ & $\begin{array}{l}97,5 \% \\
\text { cuantil }\end{array}$ \\
\hline Intercept & 0,9648 & 0,0461 & 0,8740 & 0,9339 & 0,9647 & 0,9956 & 1,0554 \\
\hline Densidad población & $-0,1755$ & 0,0029 & $-0,1811$ & $-0,1774$ & $-0,1755$ & $-0,1736$ & $-0,1699$ \\
\hline Densidad IRR & 0,8390 & 0,8344 & 0,8374 & 0,8390 & 0,8407 & 0,8437 & 0,8390 \\
\hline Tasa pesempleo & $-0,0068$ & 0,0012 & $-0,0093$ & $-0,0076$ & $-0,0067$ & $-0,0059$ & $-0,0044$ \\
\hline Tasa pesempleo IRR & 0,9932 & 0,9908 & 0,9924 & 0,9933 & 0,9941 & 0,9956 & 0,9932 \\
\hline $\begin{array}{l}\text { Porcentaje población } \\
\text { pxtranjera }\end{array}$ & 0,0013 & 0,0006 & 0,0001 & 0,0009 & 0,0013 & 0,0018 & 0,0026 \\
\hline $\begin{array}{l}\text { Porcentaje población } \\
\text { pxtranjera IRR }\end{array}$ & 1,0013 & 1,0001 & 1,0009 & 1,0013 & 1,0018 & 1,0026 & 1,0013 \\
\hline s.d.-Provincias & 0,2948 & 0,0300 & 0,2424 & 0,2734 & 0,2924 & 0,3135 & 0,3604 \\
\hline
\end{tabular}

FUENTE: Elaboración propia. 


\section{CUADRO 2.1 (continuación)}

\section{DISTRIBUCIONES A POSTERIORI DE LOS COEFICIENTES DE REGRESIÓN,} TOTAL (1999, 2005, 2009 Y 2011)

\begin{tabular}{|c|c|c|c|c|c|c|c|}
\hline \multicolumn{8}{|c|}{2011} \\
\hline & Media & s.d. & $\begin{array}{c}2,5 \% \\
\text { cuantil }\end{array}$ & $\begin{array}{c}25 \% \\
\text { cuantil }\end{array}$ & $\begin{array}{c}50 \% \\
\text { cuantil }\end{array}$ & $\begin{array}{c}75 \% \\
\text { cuantil }\end{array}$ & $\begin{array}{l}97,5 \% \\
\text { cuantil }\end{array}$ \\
\hline Intercept & 1,4225 & 0.0423 & 1,3392 & 1,3942 & 1,4225 & 1,4509 & 1,5056 \\
\hline Densidad población & $-0,1634$ & 0,0030 & $-0,1693$ & $-0,1654$ & $-0,1634$ & $-0,1614$ & $-0,1575$ \\
\hline Densidad IRR & 0,8492 & 0,8442 & 0,8475 & 0,8492 & 0,8510 & 0,8543 & 0,8492 \\
\hline Tasa desempleo & $-0,0394$ & 0,0017 & $-0,0426$ & $-0,0405$ & $-0,0394$ & $-0,0382$ & $-0,0361$ \\
\hline Tasa desempleo IRR & 0,9614 & 0,9583 & 0,9603 & 0,9614 & 0,9625 & 0,9645 & 0,9614 \\
\hline $\begin{array}{l}\text { Porcentaje población } \\
\text { extranjera }\end{array}$ & $-0,0014$ & 0,0006 & $-0,0027$ & $-0,0019$ & $-0,0014$ & $-0,0010$ & $-0,0002$ \\
\hline $\begin{array}{l}\text { Porcentaje población } \\
\text { extranjera IRR }\end{array}$ & 0,9986 & 0,9973 & 0,9981 & 0,9986 & 0,9990 & 0,9998 & 0,9986 \\
\hline s.d.-Provincias & 0,2480 & 0,0263 & 0,2021 & 0,2293 & 0,2459 & 0,2644 & 0,3053 \\
\hline
\end{tabular}

FUENTE: Elaboración propia.

tener unos índices claramente más bajos que en el caso de cajas y bancos si bien, con el tiempo, y como ocurre con el resto de instituciones financieras, los valores se van acercando a la unidad.

Por lo que se refiere a la tasa de desempleo, la primera columna del Cuadro 2.1 también muestra un efecto negativo para el conjunto de entidades bancarias, pero el signo es inestable (en 2005 pasa a ser positivo), si bien su coeficiente es bajo en valor absoluto. Los efectos son similares al reparar en cada uno de los agregados institucionales (Cuadros 2.2, 2.3 y 2.4). La explicación de este efecto negativo podría tener cierta relación con posibles desequilibrios en algunos mercados de trabajo locales que, de existir, podrían desincentivar la localización de oficinas en lugares allá donde dichos desequilibrios puedan estar presentes. De nuevo, serían las cooperativas y, en menor medida, las cajas, las entidades más indiferentes a este tipo de efecto, al mostrar unos coeficientes más bajos en valor absoluto. En este sentido, la ratio entre oficinas observadas y esperadas que nos indica la tasa de incidencia relativa (IRR) no solo nos confirmaría esta realidad sino que, además, muestra como, independientemente del tipo de entidad, los valores han ido disminuyendo desde 2005. Estas tendencias contribuirían a la exacerbación de la exclusión financiera, pues las entidades bancarias estarían abandonando aquellas regiones con niveles de desempleo más elevados.

En relación con el porcentaje de población extranjera presente en cada uno de los municipios en la muestra, como se observa en el Cuadro 2.1 (conjunto de entidades bancarias), el efecto sobre el número de oficinas bancarias esperado es, en general, de magnitud reducida aunque positiva, con un IRR en el entorno de 1 indicando un efecto reducido de esta variable en el número esperado de oficinas. Sin embargo, la 
CUADRO 2.2

DISTRIBUCIONES A POSTERIORI DE LOS COEFICIENTES DE REGRESIÓN, BANCOS (1999, 2005, 2009 Y 2011)

\begin{tabular}{|c|c|c|c|c|c|c|c|}
\hline \multicolumn{8}{|c|}{1999} \\
\hline & Media & s.d. & $\begin{array}{c}2,5 \% \\
\text { cuantil }\end{array}$ & $\begin{array}{c}25 \% \\
\text { cuantil }\end{array}$ & $\begin{array}{c}\mathbf{5 0 \%} \\
\text { cuantil }\end{array}$ & $\begin{array}{c}75 \% \\
\text { cuantil }\end{array}$ & $\begin{array}{l}97,5 \% \\
\text { cuantil }\end{array}$ \\
\hline Intercept & 0,8045 & 0,0532 & 0,6996 & 0,7688 & 0,8046 & 0,8403 & 0,9088 \\
\hline Densidad población & $-0,1164$ & 0,0050 & $-0,1263$ & $-0,1198$ & $-0,1164$ & $-0,1130$ & $-0,1065$ \\
\hline Densidad IRR & 0,8901 & 0,8813 & 0,8871 & 0,8901 & 0,8931 & 0,8989 & 0,8901 \\
\hline Tasa desempleo & $-0,0378$ & 0,0049 & $-0,0474$ & $-0,0411$ & $-0,0378$ & $-0,0345$ & $-0,0283$ \\
\hline Tasa desempleo IRR & 0,9629 & 0,9537 & 0,9597 & 0,9629 & 0,9661 & 0,9721 & 0,9629 \\
\hline $\begin{array}{l}\text { Porcentaje población } \\
\text { extranjera }\end{array}$ & 0,0142 & 0,0020 & 0,0101 & 0,0128 & 0,0142 & 0,0155 & 0,0181 \\
\hline $\begin{array}{l}\text { Porcentaje población } \\
\text { extranjera IRR }\end{array}$ & 1,0143 & 1,0102 & 1,0129 & 1,0143 & 1,0157 & 1,0183 & 1,0143 \\
\hline s.d.-Provincias & 0,2978 & 0,0317 & 0,2423 & 0,2752 & 0,2953 & 0,3177 & 0,3670 \\
\hline \multicolumn{8}{|c|}{2005} \\
\hline & Media & s.d. & $\begin{array}{c}2,5 \% \\
\text { cuantil }\end{array}$ & $\begin{array}{c}25 \% \\
\text { cuantil }\end{array}$ & $\begin{array}{c}\mathbf{5 0 \%} \\
\text { cuantil }\end{array}$ & $\begin{array}{c}75 \% \\
\text { cuantil }\end{array}$ & $\begin{array}{l}97,5 \% \\
\text { cuantil }\end{array}$ \\
\hline Intercept & 0,6638 & 0,0553 & 0,5550 & 0,6268 & 0,6638 & 0,7009 & 0,7724 \\
\hline Densidad población & $-0,1474$ & 0,0050 & $-0,1572$ & $-0,1507$ & $-0,1474$ & $-0,1440$ & $-0,1375$ \\
\hline Densidad IRR & 0,8630 & 0,8546 & 0,8601 & 0,8630 & 0,8659 & 0,8715 & 0,8630 \\
\hline Tasa pesempleo & 0,0026 & 0,0017 & $-0,0010$ & 0,0015 & 0,0027 & 0,0038 & 0,0056 \\
\hline Tasa pesempleo IRR & 1,0026 & 0,9990 & 1,0015 & 1,0027 & 1,0038 & 1,0056 & 1,0026 \\
\hline $\begin{array}{l}\text { Porcentaje población } \\
\text { extranjera }\end{array}$ & 0,0106 & 0,0013 & 0,0080 & 0,0097 & 0,0106 & 0,0115 & 0,0133 \\
\hline $\begin{array}{l}\text { Porcentaje población } \\
\text { extranjera IRR }\end{array}$ & 1,0107 & 1,0080 & 1,0098 & 1,0107 & 1,0116 & 1,0133 & 1,0107 \\
\hline s.d.-Provincias & 0,3275 & 0,0350 & 0,2663 & 0,3026 & 0,3247 & 0,3494 & 0,4039 \\
\hline \multicolumn{8}{|c|}{2009} \\
\hline & Media & s.d. & $\begin{array}{c}2,5 \% \\
\text { cuantil }\end{array}$ & $\begin{array}{c}25 \% \\
\text { cuantil }\end{array}$ & $\begin{array}{c}\mathbf{5 0 \%} \\
\text { cuantil }\end{array}$ & $\begin{array}{c}75 \% \\
\text { cuantil }\end{array}$ & $\begin{array}{l}97,5 \% \\
\text { cuantil }\end{array}$ \\
\hline Intercept & 0,6918 & 0,0582 & 0,5773 & 0,6527 & 0,6917 & 0,7308 & 0,8063 \\
\hline Densidad población & $-0,1346$ & 0,0044 & $-0,1431$ & $-0,1375$ & $-0,1346$ & $-0,1317$ & $-0,1260$ \\
\hline Densidad IRR & 0,8741 & 0,8666 & 0,8715 & 0,8741 & 0,8766 & 0,8816 & 0,8741 \\
\hline Tasa desempleo & $-0,0112$ & 0,0022 & $-0,0157$ & $-0,0127$ & $-0,0112$ & $-0,0097$ & $-0,0070$ \\
\hline Tasa desempleo IRR & 0,9888 & 0,9845 & 0,9874 & 0,9889 & 0,9903 & 0,9930 & 0,9888 \\
\hline $\begin{array}{l}\text { Porcentaje población } \\
\text { extranjera }\end{array}$ & 0,0045 & 0,0009 & 0,0026 & 0,0038 & 0,0045 & 0,0051 & 0,0063 \\
\hline $\begin{array}{l}\text { Porcentaje población } \\
\text { extranjera IRR }\end{array}$ & 1,0045 & 1,0026 & 1,0038 & 1,0045 & 1,0051 & 1,0063 & 1,0045 \\
\hline s.d.-Provincias & 0,3410 & 0,0356 & 0,2789 & 0,3156 & 0,3382 & 0,3632 & 0,4187 \\
\hline
\end{tabular}

FUENTE: Elaboración propia. 
CUADRO 2.2 (continuación)

DISTRIBUCIONES A POSTERIORI DE LOS COEFICIENTES DE REGRESIÓN, BANCOS (1999, 2005, 2009 Y 2011)

\begin{tabular}{|c|c|c|c|c|c|c|c|}
\hline \multicolumn{8}{|c|}{2011} \\
\hline & Media & s.d. & $\begin{array}{c}2,5 \% \\
\text { cuantil }\end{array}$ & $\begin{array}{c}25 \% \\
\text { cuantil }\end{array}$ & $\begin{array}{c}50 \% \\
\text { cuantil }\end{array}$ & $\begin{array}{c}75 \% \\
\text { cuantil }\end{array}$ & $\begin{array}{l}97,5 \% \\
\text { cuantil }\end{array}$ \\
\hline Intercept & 1,1151 & 0.0549 & 1,0070 & 1,0782 & 1,1152 & 1,1520 & 1,2226 \\
\hline Densidad población & $-0,1187$ & 0,0046 & $-0,1278$ & $-0,1218$ & $-0,1187$ & $-0,1156$ & $-0,1097$ \\
\hline Densidad IRR & 0,8881 & 0,8801 & 0,8853 & 0,8881 & 0,8908 & 0,8961 & 0,8881 \\
\hline Tasa desempleo & $-0,0423$ & 0,0025 & $-0,0473$ & $-0,0440$ & $-0,0423$ & $-0,0406$ & $-0,0373$ \\
\hline Tasa desempleo IRR & 0,9586 & 0,9538 & 0,9569 & 0,9586 & 0,9602 & 0,9633 & 0,9586 \\
\hline $\begin{array}{l}\text { Porcentaje población } \\
\text { extranjera }\end{array}$ & 0,0018 & 0,0010 & $-0,0001$ & 0,0012 & 0,0018 & 0,0025 & 0,0037 \\
\hline $\begin{array}{l}\text { Porcentaje población } \\
\text { extranjera IRR }\end{array}$ & 1,0018 & 0.9999 & 1,0012 & 1,0019 & 1,0025 & 1,0037 & 1,0018 \\
\hline s.d.-Provincias & 0,2857 & 0,0313 & 0,2306 & 0,2634 & 0,2834 & 0,3055 & 0,3535 \\
\hline
\end{tabular}

FUENTE: Elaboración propia.

tendencia es claramente decreciente, hasta el punto de situarse por debajo de 1 en 2011. Si suponemos que la presencia de población inmigrante es indicativo de una actividad económica más intensa, podríamos incluso suponer que, hasta cierto punto, el conjunto de empresas bancarias han llegado a sobrerreaccionar ante el estímulo que supuso una actividad económica más intensa, pues la ratio oficinas observadas/ esperadas se sitúa por encima de 1 en la mayor parte de los años analizados, sobrerreación que en el último periodo analizado habría dejado de ser tal.

El análisis por tipo de agregado institucional para esta variable (Cuadros 2.2, 2.3 y 2.4) revela diferencias que, si bien son de poca entidad, sí que ofrecen cierta consistencia. En contra de lo observado para el resto de variables analizadas, en este caso serían los bancos quienes, en todos los años analizados, presentan una tasa de incidencia relativa (IRR) por encima de la unidad, indicativo de una mayor implicación en zonas con mayor actividad económica (si, como indicábamos en el párrafo anterior, entendemos que mayores porcentajes de población extranjera podrían estar reflejando una actividad económica más intensa). Sin embargo, los coeficientes correspondientes al impacto sobre el número esperado de oficinas tienen un efecto claramente decreciente, que en el caso de cajas (Cuadro 2.3) y cooperativas (Cuadro 2.4) es negativo, esto es, a mayor porcentaje de población extranjera el número esperado de cajas y cooperativas es menor. Habría que tener en cuenta, sin embargo, que el efecto no es constante y, en el caso concreto de las cajas, el valor del IRR asociado a esta variable se ha ido acercando a lo largo de los años al de los bancos, fenómeno que podría venir explicado por las políticas expansivas de estas entidades en busca de territorios más dinámicos (Pastor et al., 2017). 
CUADRO 2.3

DISTRIBUCIONES A POSTERIORI DE LOS COEFICIENTES DE REGRESIÓN, CAJAS DE AHORROS (1999, 2005, 2009 Y 2011)

\begin{tabular}{|c|c|c|c|c|c|c|c|}
\hline \multicolumn{8}{|c|}{1999} \\
\hline & Media & s.d. & $\begin{array}{c}2,5 \% \\
\text { cuantil }\end{array}$ & $\begin{array}{l}25 \% \\
\text { cuantil }\end{array}$ & $\begin{array}{c}50 \% \\
\text { cuantil }\end{array}$ & $\begin{array}{c}75 \% \\
\text { cuantil }\end{array}$ & $\begin{array}{l}97,5 \% \\
\text { cuantil }\end{array}$ \\
\hline Intercept & 1,2078 & 0.0594 & 1,0904 & 1,1681 & 1,2080 & 1,2477 & 1,3240 \\
\hline Densidad población & $-0,1787$ & 0,0050 & $-0,1884$ & $-0,1821$ & $-0,1787$ & $-0,1754$ & $-0,1690$ \\
\hline Densidad IRR & 0,8363 & 0,8282 & 0,8335 & 0,8363 & 0,8392 & 0,8445 & 0,8363 \\
\hline Tasa desempleo & $-0,0471$ & 0,0049 & $-0,0566$ & $-0,0504$ & $-0,0471$ & $-0,0438$ & $-0,0375$ \\
\hline Tasa desempleo IRR & 0,9540 & 0,9449 & 0,9509 & 0,9540 & 0,9572 & 0,9632 & 0,9540 \\
\hline $\begin{array}{l}\text { Porcentaje población } \\
\text { extranjera }\end{array}$ & $-0,0042$ & 0,0026 & $-0,0094$ & $-0,0060$ & $-0,0042$ & $-0,0025$ & 0,0008 \\
\hline $\begin{array}{l}\text { Porcentaje población } \\
\text { extranjera IRR }\end{array}$ & 0,9958 & 0,9907 & 0,9941 & 0,9958 & 0,9975 & 1,0008 & 0,9958 \\
\hline s.d.-Provincias & 0,3555 & 0,0375 & 0,2901 & 0,3288 & 0,3525 & 0,3789 & 0,4374 \\
\hline \multicolumn{8}{|c|}{2005} \\
\hline & Media & s.d. & $\begin{array}{c}2,5 \% \\
\text { cuantil }\end{array}$ & $\begin{array}{c}25 \% \\
\text { cuantil }\end{array}$ & $\begin{array}{c}\mathbf{5 0 \%} \\
\text { cuantil }\end{array}$ & $\begin{array}{c}75 \% \\
\text { cuantil }\end{array}$ & $\begin{array}{l}97,5 \% \\
\text { cuantil }\end{array}$ \\
\hline Intercept & 0,9610 & 0,0569 & 0,8489 & 0,9229 & 0,9611 & 0,9992 & 1,0728 \\
\hline Densidad población & $-0,1797$ & 0,0044 & $-0,1883$ & $-0,1827$ & $-0,1798$ & $-0,1768$ & $-0,1712$ \\
\hline Densidad IRR & 0,8355 & 0,8284 & 0,8330 & 0,8355 & 0,8379 & 0,8427 & 0,8355 \\
\hline Tasa desempleo & $-0,0035$ & 0,0028 & $-0,0096$ & $-0,0053$ & $-0,0033$ & $-0,0015$ & 0,0015 \\
\hline Tasa desempleo IRR & 0,9965 & 0,9904 & 0,9947 & 0,9967 & 0,9985 & 1,0015 & 0,9965 \\
\hline $\begin{array}{l}\text { Porcentaje población } \\
\text { extranjera }\end{array}$ & $-0,0015$ & 0,0013 & $-0,0041$ & $-0,0024$ & $-0,0015$ & $-0,0006$ & 0,0011 \\
\hline $\begin{array}{l}\text { Porcentaje población } \\
\text { extranjera IRR }\end{array}$ & 0,9985 & 0,9959 & 0,9976 & 0,9985 & 0,9994 & 1,0011 & 0,9985 \\
\hline s.d.-Provincias & 0,3511 & 0,0366 & 0,2871 & 0,3250 & 0,3482 & 0,3740 & 0,4311 \\
\hline \multicolumn{8}{|c|}{2009} \\
\hline & Media & s.d. & $\begin{array}{c}2,5 \% \\
\text { cuantil }\end{array}$ & $\begin{array}{c}25 \% \\
\text { cuantil }\end{array}$ & $\begin{array}{c}50 \% \\
\text { cuantil }\end{array}$ & $\begin{array}{c}75 \% \\
\text { cuantil }\end{array}$ & $\begin{array}{l}97,5 \% \\
\text { cuantil }\end{array}$ \\
\hline Intercept & 0,9398 & 0,0573 & 0,8269 & 0,9013 & 0,9397 & 0,9782 & 1,0526 \\
\hline Densidad población & $-0,1783$ & 0,0042 & $-0,1865$ & $-0,1811$ & $-0,1783$ & $-0,1755$ & $-0,1701$ \\
\hline Densidad IRR & 0,8367 & 0,8299 & 0,8344 & 0,8367 & 0,8391 & 0,8436 & 0,8367 \\
\hline Tasa desempleo & $-0,0038$ & 0,0015 & $-0,0068$ & $-0,0047$ & $-0,0037$ & $-0,0028$ & $-0,0011$ \\
\hline Tasa desempleo IRR & 0,9962 & 0,9932 & 0,9953 & 0,9963 & 0,9972 & 0,9989 & 0,9962 \\
\hline $\begin{array}{l}\text { Porcentaje población } \\
\text { extranjera }\end{array}$ & $-0,0002$ & 0,0010 & $-0,0021$ & $-0,0008$ & $-0,0002$ & 0,0005 & 0,0017 \\
\hline $\begin{array}{l}\text { Porcentaje población } \\
\text { extranjera IRR }\end{array}$ & 0,9998 & 0,9979 & 0,9992 & 0,9998 & 1,0005 & 1,0017 & 0,9998 \\
\hline s.d.-Provincias & 0,3571 & 0,0368 & 0,2928 & 0,3308 & 0,3541 & 0,3801 & 0,4376 \\
\hline
\end{tabular}

FUENTE: Elaboración propia. 


\section{CUADRO 2.3 (continuación)}

\section{DISTRIBUCIONES A POSTERIORI DE LOS COEFICIENTES DE REGRESIÓN,} CAJAS DE AHORROS (1999, 2005, 2009 Y 2011)

\begin{tabular}{|c|c|c|c|c|c|c|c|}
\hline \multicolumn{8}{|c|}{2011} \\
\hline & Media & s.d. & $\begin{array}{l}2,5 \% \\
\text { cuantil }\end{array}$ & $\begin{array}{l}25 \% \\
\text { cuantil }\end{array}$ & $\begin{array}{l}50 \% \\
\text { cuantil }\end{array}$ & $\begin{array}{l}75 \% \\
\text { cuantil }\end{array}$ & $\begin{array}{l}97,5 \% \\
\text { cuantil }\end{array}$ \\
\hline Intercept & 1,4259 & 0,0597 & 1,3083 & 1,3858 & 1,4259 & 1,4660 & 1,5431 \\
\hline Densidad población & $-0,1709$ & 0,0044 & $-0,1795$ & $-0,1739$ & $-0,1709$ & $-0,1679$ & $-0,1622$ \\
\hline Densidad IRR & 0,8429 & 0,8357 & 0,8404 & 0,8429 & 0,8454 & 0,8503 & 0,8429 \\
\hline Tasa desempleo & $-0,0373$ & 0,0024 & $-0,0421$ & $-0,0390$ & $-0,0373$ & $-0,0357$ & $-0,0326$ \\
\hline Tasa desempleo IRR & 0,9633 & 0,9588 & 0,9618 & 0,9633 & 0,9649 & 0,9679 & 0,9633 \\
\hline $\begin{array}{l}\text { Porcentaje población } \\
\text { extranjera }\end{array}$ & $-0,0033$ & 0,0010 & $-0,0052$ & $-0,0039$ & $-0,0033$ & $-0,0026$ & $-0,0014$ \\
\hline $\begin{array}{l}\text { Porcentaje población } \\
\text { extranjera IRR }\end{array}$ & 0,9967 & 0,9948 & 0,9961 & 0,9967 & 0,9974 & 0,9986 & 0,9967 \\
\hline s.d.-Provincias & 0,3439 & 0,0364 & 0,2802 & 0,3179 & 0,3410 & 0,3667 & 0,4235 \\
\hline
\end{tabular}

FUENTE: Elaboración propia.

El impacto que la presencia de otro tipo de entidades bancarias puede tener sobre el resto de agregados institucionales vendría recogido en los Cuadros 2.5, 2.7 y 2.8 para bancos, cajas y cooperativas, respectivamente. En el primer caso (Cuadro 2.5) vendría recogido cuál sería el efecto que tiene la presencia de oficinas de cajas y cooperativas sobre el número esperado de oficinas de bancos, y los otros dos cuadros deberían ser interpretados de forma análoga. Si bien los resultados no son del todo claros, una de las tendencias observadas es que, en el caso de las cajas (Cuadro 2.6), es más probable que se ubiquen en un lugar con presencia de cooperativas que si hay oficinas de bancos, pero si comparamos 1999 y 2011 se observa que las tendencias son opuestas, esto es, durante el periodo parecen haber ido abandonando lugares con mayor densidad de cooperativas para ir optando por otros con más oficinas de bancos. Por tanto, se confirmaría el efecto ya apuntado en Alamá et al. (2015) de cierto efecto sustitutivo, entre cajas y cooperativas pues que la presencia de estas últimas podría ser un elemento que, con el tiempo, tendría un impacto más débil sobre la posibilidad de que una caja se expanda por un territorio, siendo el efecto de la presencia de bancos el opuesto. Estas tendencias darían validez, además, a las teorías sobre precedencia rival y seguimiento racional (rational herding, Chang et $a l .$, 1997), que podrían así estar presentes en el sector bancario español, si bien no para todas las entidades.

Una vez considerados los efectos de todas las covariables, los Gráficos 2.1-2.4 ofrecen una ilustración del IRR propio de cada provincia, que correspondería a los distintos tipos de entidades consideradas. Cada uno de ellos se divide en cuatro paneles de acuerdo con los periodos analizados. 


\section{CUADRO 2.4}

DISTRIBUCIONES A POSTERIORI DE LOS COEFICIENTES DE REGRESIÓN, COOPERATIVAS DE CRÉDITO $(1999,2005,2009$ Y 2011)

\begin{tabular}{|c|c|c|c|c|c|c|c|}
\hline \multicolumn{8}{|c|}{1999} \\
\hline & Media & s.d. & $\begin{array}{c}2,5 \% \\
\text { cuantil }\end{array}$ & $\begin{array}{c}25 \% \\
\text { cuantil }\end{array}$ & $\begin{array}{c}\mathbf{5 0 \%} \\
\text { cuantil }\end{array}$ & $\begin{array}{c}75 \% \\
\text { cuantil }\end{array}$ & $\begin{array}{l}97,5 \% \\
\text { cuantil }\end{array}$ \\
\hline Intercept & 2,1536 & 0,1611 & 1,8330 & 2,0466 & 2,1547 & 2,2618 & 2,4677 \\
\hline Densidad población & $-0,3873$ & 0,0113 & $-0,4095$ & $-0,3950$ & $-0,3873$ & $-0,3797$ & $-0,3651$ \\
\hline Densidad IRR & 0,6789 & 0,6639 & 0,6737 & 0,6789 & 0,6841 & 0,6941 & 0,6789 \\
\hline Tasa desempleo & $-0,0577$ & 0,0092 & $-0,0758$ & $-0,0639$ & $-0,0577$ & $-0,0515$ & $-0,0397$ \\
\hline Tasa desempleo IRR & 0,9440 & 0,9270 & 0,9381 & 0,9440 & 0,9498 & 0,9610 & 0,9440 \\
\hline $\begin{array}{l}\text { Porcentaje población } \\
\text { extranjera }\end{array}$ & $-0,0109$ & 0,0056 & $-0,0223$ & $-0,0146$ & $-0,0108$ & $-0,0070$ & $-0,0002$ \\
\hline $\begin{array}{l}\text { Porcentaje población } \\
\text { extranjera IRR }\end{array}$ & 0,9892 & 0,9780 & 0,9855 & 0,9893 & 0,9930 & 0,9998 & 0,9892 \\
\hline s.d.-Provincias & 1,0455 & 0,1149 & 0,8441 & 0,9626 & 1,0352 & 1,1163 & 1,2957 \\
\hline \multicolumn{8}{|c|}{2005} \\
\hline & Media & s.d. & $\begin{array}{c}2.5 \% \\
\text { cuantil }\end{array}$ & $\begin{array}{c}25 \% \\
\text { cuantil }\end{array}$ & $\begin{array}{c}\mathbf{5 0 \%} \\
\text { cuantil }\end{array}$ & $\begin{array}{c}75 \% \\
\text { cuantil }\end{array}$ & $\begin{array}{l}97.5 \% \\
\text { cuantil }\end{array}$ \\
\hline Intercept & 1,7857 & 0.1466 & 1,4947 & 1,6883 & 1,7865 & 1,8840 & 2,0724 \\
\hline Densidad población & $-0,3556$ & 0,0091 & $-0,3735$ & $-0,3617$ & $-0,3556$ & $-0,3494$ & $-0,3377$ \\
\hline Densidad IRR & 0,7008 & 0,6883 & 0,6965 & 0,7008 & 0,7051 & 0,7134 & 0,7008 \\
\hline Tasa desempleo & 0,0012 & 0,0024 & $-0,0041$ & $-0,0003$ & 0,0014 & 0,0029 & 0,0054 \\
\hline Tasa desempleo IRR & 1,0012 & 0,9959 & 0,9997 & 1,0014 & 1,0029 & 1,0054 & 1,0012 \\
\hline $\begin{array}{l}\text { Porcentaje población } \\
\text { extranjera }\end{array}$ & $-0,0057$ & 0,0026 & $-0,0109$ & $-0,0075$ & $-0,0057$ & $-0,0039$ & $-0,0006$ \\
\hline $\begin{array}{l}\text { Porcentaje población } \\
\text { extranjera IRR }\end{array}$ & 0,9943 & 0,9891 & 0,9926 & 0,9943 & 0,9961 & 0,9994 & 0,9943 \\
\hline s.d.-Provincias & 0,9712 & 0,1054 & 0,7872 & 0,8960 & 0,9627 & 1,0370 & 1,2014 \\
\hline \multicolumn{8}{|c|}{2009} \\
\hline & Media & s.d. & $\begin{array}{c}2.5 \% \\
\text { cuantil }\end{array}$ & $\begin{array}{c}25 \% \\
\text { cuantil }\end{array}$ & $\begin{array}{c}50 \% \\
\text { cuantil }\end{array}$ & $\begin{array}{c}75 \% \\
\text { cuantil }\end{array}$ & $\begin{array}{l}97.5 \% \\
\text { cuantil }\end{array}$ \\
\hline Intercept & 1,7475 & 0,1426 & 1,4650 & 1,6525 & 1,7480 & 1,8431 & 2,0268 \\
\hline Densidad Población & $-0,3315$ & 0,0089 & $-0,3490$ & $-0,3375$ & $-0,3315$ & $-0,3255$ & $-0,3140$ \\
\hline Densidad IRR & 0,7178 & 0,7054 & 0,7135 & 0,7178 & 0,7222 & 0,7305 & 0,7178 \\
\hline Tasa desempleo & $-0,0032$ & 0,0032 & $-0,0103$ & $-0,0052$ & $-0,0030$ & $-0,0010$ & 0,0023 \\
\hline Tasa desempleo IRR & 0,9968 & 0,9898 & 0,9948 & 0,9970 & 0,9990 & 1,0023 & 0,9968 \\
\hline $\begin{array}{l}\text { Porcentaje población } \\
\text { extranjera }\end{array}$ & $-0,0073$ & 0,0021 & $-0,0114$ & $-0,0087$ & $-0,0073$ & $-0,0059$ & $-0,0033$ \\
\hline $\begin{array}{l}\text { Porcentaje población } \\
\text { extranjera IRR }\end{array}$ & 0,9927 & 0,9887 & 0,9913 & 0,9927 & 0,9941 & 0,9967 & 0,9927 \\
\hline s.d.-Provincias & 0,9275 & 0,1004 & 0,7522 & 0,8559 & 0,9194 & 0,9902 & 1,1469 \\
\hline
\end{tabular}

FUENTE: Elaboración propia. 
CUADRO 2.4 (continuación)

DISTRIBUCIONES A POSTERIORI DE LOS COEFICIENTES DE REGRESIÓN, COOPERATIVAS DE CRÉDITO (1999, 2005, 2009 Y 2011)

\begin{tabular}{|c|c|c|c|c|c|c|c|}
\hline \multicolumn{8}{|c|}{2011} \\
\hline & Media & s.d. & $\begin{array}{l}2,5 \% \\
\text { cuantil }\end{array}$ & $\begin{array}{l}25 \% \\
\text { cuantil }\end{array}$ & $\begin{array}{l}50 \% \\
\text { cuantil }\end{array}$ & $\begin{array}{l}75 \% \\
\text { cuantil }\end{array}$ & $\begin{array}{l}97,5 \% \\
\text { cuantil }\end{array}$ \\
\hline Intercept & 2,0550 & 0,1462 & 1,7654 & 1,9574 & 2,0555 & 2,1531 & 2,3412 \\
\hline Densidad población & $-0,3161$ & 0,0095 & $-0,3348$ & $-0,3225$ & $-0,3161$ & $-0,3096$ & $-0,2973$ \\
\hline Densidad IRR & 0,7290 & 0,7155 & 0,7243 & 0,7290 & 0,7337 & 0,7428 & 0,7290 \\
\hline Tasa desempleo & $-0,0279$ & 0,0049 & $-0,0375$ & $-0,0312$ & $-0,0279$ & $-0,0246$ & $-0,0184$ \\
\hline Tasa desempleo IRR & 0,9725 & 0,9632 & 0,9693 & 0,9725 & 0,9757 & 0,9818 & 0,9725 \\
\hline $\begin{array}{l}\text { Porcentaje población } \\
\text { extranjera }\end{array}$ & $-0,0076$ & 0,0020 & $-0,0116$ & $-0,0089$ & $-0,0076$ & $-0,0062$ & $-0,0036$ \\
\hline $\begin{array}{l}\text { Porcentaje población } \\
\text { extranjera IRR }\end{array}$ & 0,9925 & 0,9885 & 0,9911 & 0,9925 & 0,9938 & 0,9964 & 0,9925 \\
\hline s.d.-Provincias & 0,9161 & 0,0982 & 0,7444 & 0,8460 & 0,9082 & 0,9774 & 1,1306 \\
\hline
\end{tabular}

FUENTE: Elaboración propia.

Las diferencias observadas más importantes se dan al comparar los agregados institucionales y, en concreto, y en línea con los resultados correspondientes a los Cuadros 2.1-2.4 ya comentados en los párrafos anteriores, existen más semejanzas cuando comparamos las tendencias observadas para bancos y cajas de ahorros (Gráficos 2.2 y 2.3 , respectivamente) que cuando comparamos cualesquiera de estos dos agregados institucionales con las cooperativas (Gráfico 2.4), independientemente del año considerado. Si bien en los dos primeros casos se aprecia que, para muchos años y muchas provincias, los valores de IRR se corresponderían más bien con una escasez relativa de oficinas (particularmente en la mitad sur del país), en el caso de las cooperativas se observa lo que podría ser un exceso de oficinas, especialmente en gran parte de la costa mediterránea (a excepción de Cataluña), si bien se trataría de una tendencia que ha ido cambiando ligeramente entre 1999 y 2011. Por tanto, ante la todavía pendiente reestructuración en el sector de las cooperativas de crédito, se trataría este de un análisis que podría contribuir positivamente al rediseño de la red de oficinas en España.

En el caso de bancos y cajas, el fenómeno es de carácter opuesto y estamos siempre mucho más cerca de una escasez de oficinas, pues muchas provincias, de nuevo pertenecientes a la mitad sur de España, tendrían valores por debajo de la unidad. Por tanto, pese a la intensa reestructuración en el sector de las cajas de ahorros que ha tenido lugar desde el inicio de la crisis (como se puede observar al comparar los distintos paneles del Gráfico 2.3), habría numerosas áreas en las que el acceso a los servicios bancarios a través de la oficina física sería claramente mejorable. 


\section{CUADRO 2.5}

DISTRIBUCIONES A POSTERIORI DE LOS COEFICIENTES DE REGRESIÓN, EFECTO DE OTRO TIPO DE ENTIDADES, BANCOS (1999, 2005, 2009 Y 2011)

\begin{tabular}{|c|c|c|c|c|c|c|c|}
\hline \multicolumn{8}{|c|}{1999} \\
\hline & Media & s.d. & $\begin{array}{c}2,5 \% \\
\text { cuantil }\end{array}$ & $\begin{array}{c}25 \% \\
\text { cuantil }\end{array}$ & $\begin{array}{c}50 \% \\
\text { cuantil }\end{array}$ & $\begin{array}{c}75 \% \\
\text { cuantil }\end{array}$ & $\begin{array}{l}97,5 \% \\
\text { cuantil }\end{array}$ \\
\hline Cajas de ahorro & -0.0002 & 0.0000 & -0.0003 & -0.0003 & -0.0002 & -0.0002 & -0.0002 \\
\hline $\begin{array}{l}\text { Cajas de ahorro } \\
\text { IRR }\end{array}$ & 0.9998 & 0.9997 & 0.9997 & 0.9998 & 0.9998 & 0.9998 & 0.9998 \\
\hline $\begin{array}{l}\text { Cooperativas de } \\
\text { crédito }\end{array}$ & 0.0563 & 0.0010 & 0.0542 & 0.0556 & 0.0563 & 0.0570 & 0.0583 \\
\hline $\begin{array}{l}\text { Cooperativas de } \\
\text { crédito IRR }\end{array}$ & 1,0579 & 1,0557 & 1,0571 & 1,0579 & 1,0586 & 1,0600 & 1,0579 \\
\hline \multicolumn{8}{|c|}{2005} \\
\hline & Media & s.d. & $\begin{array}{c}2,5 \% \\
\text { cuantil }\end{array}$ & $\begin{array}{c}25 \% \\
\text { cuantil }\end{array}$ & $\begin{array}{c}50 \% \\
\text { cuantil }\end{array}$ & $\begin{array}{c}75 \% \\
\text { cuantil }\end{array}$ & $\begin{array}{l}97,5 \% \\
\text { cuantil }\end{array}$ \\
\hline Cajas de ahorro & $-0,0004$ & 0,0000 & $-0,0005$ & $-0,0004$ & $-0,0004$ & $-0,0004$ & $-0,0003$ \\
\hline $\begin{array}{l}\text { Cajas de ahorro } \\
\text { IRR }\end{array}$ & 0,9996 & 0,9995 & 0,9996 & 0,9996 & 0,9996 & 0,9997 & 0,9996 \\
\hline $\begin{array}{l}\text { Cooperativas de } \\
\text { crédito }\end{array}$ & 0,0346 & 0,0007 & 0,0331 & 0,0341 & 0,0346 & 0,0351 & 0,0360 \\
\hline $\begin{array}{l}\text { Cooperativas de } \\
\text { crédito IRR }\end{array}$ & 1,0352 & 1,0337 & 1,0347 & 1,0352 & 1,0357 & 1,0367 & 1,0352 \\
\hline \multicolumn{8}{|c|}{2009} \\
\hline & Media & s.d. & $\begin{array}{l}2,5 \% \\
\text { cuantil }\end{array}$ & $\begin{array}{c}25 \% \\
\text { cuantil }\end{array}$ & $\begin{array}{c}50 \% \\
\text { cuantil }\end{array}$ & $\begin{array}{c}75 \% \\
\text { cuantil }\end{array}$ & $\begin{array}{l}97,5 \% \\
\text { cuantil }\end{array}$ \\
\hline Cajas de ahorro & $-0,0004$ & 0,0000 & $-0,0005$ & $-0,0004$ & $-0,0004$ & $-0,0004$ & $-0,0004$ \\
\hline $\begin{array}{l}\text { Cajas de ahorro } \\
\text { IRR }\end{array}$ & 0,9996 & 0,9995 & 0,9996 & 0,9996 & 0,9996 & 0,9996 & 0,9996 \\
\hline $\begin{array}{l}\text { Cooperativas de } \\
\text { crédito }\end{array}$ & 0,0345 & 0,0005 & 0,0335 & 0,0342 & 0,0345 & 0,0349 & 0,0355 \\
\hline $\begin{array}{l}\text { Cooperativas de } \\
\text { crédito IRR }\end{array}$ & 1,0351 & 1,0341 & 1,0348 & 1,0351 & 1,0355 & 1,0362 & 1,0351 \\
\hline \multicolumn{8}{|c|}{2011} \\
\hline & Media & sd & $\begin{array}{c}2.5 \% \\
\text { cuantil }\end{array}$ & $\begin{array}{c}25 \% \\
\text { cuantil }\end{array}$ & $\begin{array}{c}50 \% \\
\text { cuantil }\end{array}$ & $\begin{array}{c}75 \% \\
\text { cuantil }\end{array}$ & $\begin{array}{l}97.5 \% \\
\text { cuantil }\end{array}$ \\
\hline Cajas de ahorro & $-0,0003$ & 0,0000 & $-0,0003$ & $-0,0003$ & $-0,0003$ & $-0,0002$ & $-0,0002$ \\
\hline $\begin{array}{l}\text { Cajas de ahorro } \\
\text { IRR }\end{array}$ & 0,9997 & 0,9997 & 0,9997 & 0,9997 & 0,9998 & 0,9998 & 0,9997 \\
\hline $\begin{array}{l}\text { Cooperativas de } \\
\text { crédito }\end{array}$ & 0,0340 & 0,0006 & 0,0328 & 0,0336 & 0,0340 & 0,0344 & 0,0352 \\
\hline $\begin{array}{l}\text { Cooperativas de } \\
\text { crédito IRR }\end{array}$ & 1,0346 & 1,0334 & 1,0342 & 1,0346 & 1,0350 & 1,0358 & 1,0346 \\
\hline
\end{tabular}

FUENTE: Elaboración propia. 
CUADRO 2.6

DISTRIBUCIONES A POSTERIORI DE LOS COEFICIENTES DE REGRESIÓN, EFECTO DE OTRO TIPO DE ENTIDADES, CAJAS DE AHORROS $(1999,2005,2009$ Y 2011)

\begin{tabular}{|c|c|c|c|c|c|c|c|}
\hline \multicolumn{8}{|c|}{1999} \\
\hline & Media & s.d. & $\begin{array}{c}2,5 \% \\
\text { cuantil }\end{array}$ & $\begin{array}{c}25 \% \\
\text { cuantil }\end{array}$ & $\begin{array}{l}50 \% \\
\text { cuantil }\end{array}$ & $\begin{array}{c}75 \% \\
\text { cuantil }\end{array}$ & $\begin{array}{l}97,5 \% \\
\text { cuantil }\end{array}$ \\
\hline Bancos comerciales & $-0,0001$ & 0,0000 & $-0,0002$ & $-0,0002$ & $-0,0001$ & $-0,0001$ & $-0,0001$ \\
\hline $\begin{array}{l}\text { Bancos comerciales } \\
\text { IRR }\end{array}$ & 0,9999 & 0,9998 & 0,9998 & 0,9999 & 0,9999 & 0,9999 & 0,9999 \\
\hline $\begin{array}{l}\text { Cooperativas de } \\
\text { crédito }\end{array}$ & 0,0472 & 0,0010 & 0,0453 & 0,0465 & 0,0472 & 0,0478 & 0,0491 \\
\hline $\begin{array}{l}\text { Cooperativas de } \\
\text { crédito IRR }\end{array}$ & 1,0483 & 1,0463 & 1,0476 & 1,0483 & 1,0490 & 1,0503 & 1,0483 \\
\hline \multicolumn{8}{|c|}{2005} \\
\hline & Media & s.d. & $\begin{array}{c}2,5 \% \\
\text { cuantil }\end{array}$ & $\begin{array}{c}25 \% \\
\text { cuantil }\end{array}$ & $\begin{array}{c}50 \% \\
\text { cuantil }\end{array}$ & $\begin{array}{c}75 \% \\
\text { cuantil }\end{array}$ & $\begin{array}{l}97,5 \% \\
\text { cuantil }\end{array}$ \\
\hline Bancos comerciales & $-0,0006$ & 0,0000 & $-0,0007$ & $-0,0007$ & $-0,0006$ & $-0,0006$ & $-0,0006$ \\
\hline $\begin{array}{l}\text { Bancos comerciales } \\
\text { IRR }\end{array}$ & 0,9994 & 0,9993 & 0,9993 & 0,9994 & 0,9994 & 0,9994 & 0,9994 \\
\hline $\begin{array}{l}\text { Cooperativas de } \\
\text { crédito }\end{array}$ & 0,0342 & 0,0006 & 0,0330 & 0,0338 & 0,0342 & 0,0347 & 0,0355 \\
\hline $\begin{array}{l}\text { Cooperativas de } \\
\text { crédito IRR }\end{array}$ & 1,0348 & 1,0355 & 1,0344 & 1,0348 & 1,0353 & 1,0361 & 1,0348 \\
\hline \multicolumn{8}{|c|}{2009} \\
\hline & Media & s.d. & $\begin{array}{c}2,5 \% \\
\text { cuantil }\end{array}$ & $\begin{array}{c}25 \% \\
\text { cuantil }\end{array}$ & $\begin{array}{c}50 \% \\
\text { cuantil }\end{array}$ & $\begin{array}{c}75 \% \\
\text { cuantil }\end{array}$ & $\begin{array}{l}97,5 \% \\
\text { cuantil }\end{array}$ \\
\hline Bancos comerciales & $-0,0004$ & 0,0000 & $-0,0005$ & $-0,0004$ & $-0,0004$ & $-0,0004$ & $-0,0004$ \\
\hline $\begin{array}{l}\text { Bancos comerciales } \\
\text { IRR }\end{array}$ & 0,9996 & 0,9995 & 0,9996 & 0,9996 & 0,9996 & 0,9996 & 0,9996 \\
\hline $\begin{array}{l}\text { Cooperativas de } \\
\text { crédito }\end{array}$ & 0,0318 & 0,0005 & 0,0308 & 0,0314 & 0,0318 & 0,0321 & 0,0328 \\
\hline $\begin{array}{l}\text { Cooperativas de } \\
\text { crédito IRR }\end{array}$ & 1,0323 & 1,0312 & 1,0319 & 1,0323 & 1,0327 & 1,0333 & 1,0323 \\
\hline \multicolumn{8}{|c|}{2011} \\
\hline & Media & s.d. & $\begin{array}{c}2,5 \% \\
\text { cuantil }\end{array}$ & $\begin{array}{c}25 \% \\
\text { cuantil }\end{array}$ & $\begin{array}{c}50 \% \\
\text { cuantil }\end{array}$ & $\begin{array}{c}75 \% \\
\text { cuantil }\end{array}$ & $\begin{array}{l}97,5 \% \\
\text { cuantil }\end{array}$ \\
\hline Bancos comerciales & $-0,0003$ & 0,0000 & $-0,0004$ & $-0,0003$ & $-0,0003$ & $-0,0003$ & $-0,0003$ \\
\hline $\begin{array}{l}\text { Bancos comerciales } \\
\text { IRR }\end{array}$ & 0,9997 & 0,9996 & 0,9997 & 0,9997 & 0,9997 & 0,9997 & 0,9997 \\
\hline $\begin{array}{l}\text { Cooperativas de } \\
\text { crédito }\end{array}$ & 0,0335 & 0,0005 & 0,0324 & 0,0331 & 0,0335 & 0,0339 & 0,0345 \\
\hline $\begin{array}{l}\text { Cooperativas de } \\
\text { crédito IRR }\end{array}$ & 1,0341 & 1,0330 & 1,0337 & 1,0341 & 1,0344 & 1,0351 & 1,0341 \\
\hline
\end{tabular}

FUENTE: Elaboración propia. 
CUADRO 2.7

DISTRIBUCIONES A POSTERIORI DE LOS COEFICIENTES DE REGRESIÓN, EFECTO DE OTRO TIPO DE ENTIDADES, COOPERATIVAS DE CRÉDITO $(1999,2005,2009$ Y 2011)

\begin{tabular}{|c|c|c|c|c|c|c|c|}
\hline \multicolumn{8}{|c|}{1999} \\
\hline & Media & s.d. & $\begin{array}{c}2,5 \% \\
\text { cuantil }\end{array}$ & $\begin{array}{c}25 \% \\
\text { cuantil }\end{array}$ & $\begin{array}{c}50 \% \\
\text { cuantil }\end{array}$ & $\begin{array}{c}75 \% \\
\text { cuantil }\end{array}$ & $\begin{array}{l}97,5 \% \\
\text { cuantil }\end{array}$ \\
\hline Bancos comerciales & 0,0023 & 0,0007 & 0,0010 & 0,0018 & 0,0023 & 0,0028 & 0,0038 \\
\hline $\begin{array}{l}\text { Bancos comerciales } \\
\text { IRR }\end{array}$ & 1,0023 & 1,0010 & 1,0018 & 1,0023 & 1,0028 & 1,0038 & 1,0023 \\
\hline Cajas de ahorro & $-0,0001$ & 0,0006 & $-0,0013$ & $-0,0005$ & $-0,0001$ & 0,0003 & 0,0010 \\
\hline $\begin{array}{l}\text { Cajas de ahorro } \\
\text { IRR }\end{array}$ & 0,9999 & 0,9987 & 0,9995 & 0,9999 & 1,0003 & 1,0010 & 0,9999 \\
\hline \multicolumn{8}{|c|}{2005} \\
\hline & Media & s.d. & $\begin{array}{c}2,5 \% \\
\text { cuantil }\end{array}$ & $\begin{array}{c}25 \% \\
\text { cuantil }\end{array}$ & $\begin{array}{c}\mathbf{5 0 \%} \\
\text { cuantil }\end{array}$ & $\begin{array}{c}75 \% \\
\text { cuantil }\end{array}$ & $\begin{array}{l}97,5 \% \\
\text { cuantil }\end{array}$ \\
\hline Bancos comerciales & $-0,0002$ & 0,0004 & $-0,0010$ & $-0,0005$ & $-0,0002$ & 0,0001 & 0,0007 \\
\hline $\begin{array}{l}\text { Bancos comerciales } \\
\text { IRR }\end{array}$ & 0,9998 & 0,9990 & 0,9995 & 0,9998 & 1,0001 & 1,0007 & 0,9998 \\
\hline Cajas de ahorro & 0,0018 & 0,0004 & 0,0010 & 0,0015 & 0,0018 & 0,0020 & 0,0025 \\
\hline $\begin{array}{l}\text { Cajas de ahorro } \\
\text { IRR }\end{array}$ & 1,0018 & 1,0010 & 1,0015 & 1,0018 & 1,0020 & 1,0025 & 1,0018 \\
\hline \multicolumn{8}{|c|}{2009} \\
\hline & Media & s.d. & $\begin{array}{l}2.5 \% \\
\text { cuantil }\end{array}$ & $\begin{array}{l}25 \% \\
\text { cuantil }\end{array}$ & $\begin{array}{c}\mathbf{5 0 \%} \\
\text { cuantil }\end{array}$ & $\begin{array}{c}75 \% \\
\text { cuantil }\end{array}$ & $\begin{array}{l}97.5 \% \\
\text { cuantil }\end{array}$ \\
\hline Bancos comerciales & $-0,0032$ & 0,0004 & $-0,0041$ & $-0,0035$ & $-0,0032$ & $-0,0029$ & $-0,0024$ \\
\hline $\begin{array}{l}\text { Bancos comerciales } \\
\text { IRR }\end{array}$ & 0,9968 & 0,9959 & 0,9965 & 0,9968 & 0,9971 & 0,9976 & 0,9968 \\
\hline Cajas de ahorro & 0,0053 & 0,0005 & 0,0042 & 0,0049 & 0,0053 & 0,0056 & 0,0063 \\
\hline $\begin{array}{l}\text { Cajas de ahorro } \\
\text { IRR }\end{array}$ & 1,0053 & 1,0042 & 1,0049 & 1,0053 & 1,0056 & 1,0063 & 1,0053 \\
\hline \multicolumn{8}{|c|}{2011} \\
\hline & Media & s.d. & $\begin{array}{c}2,5 \% \\
\text { cuantil }\end{array}$ & $\begin{array}{c}25 \% \\
\text { cuantil }\end{array}$ & $\begin{array}{c}50 \% \\
\text { cuantil }\end{array}$ & $\begin{array}{c}75 \% \\
\text { cuantil }\end{array}$ & $\begin{array}{l}97,5 \% \\
\text { cuantil }\end{array}$ \\
\hline Bancos comerciales & $-0,0050$ & 0,0004 & $-0,0057$ & $-0,0052$ & $-0,0050$ & $-0,0047$ & $-0,0042$ \\
\hline $\begin{array}{l}\text { Bancos comerciales } \\
\text { IRR }\end{array}$ & 0,9950 & 0,9943 & 0,9948 & 0,9950 & 0,9953 & 0,9958 & 0,9950 \\
\hline Cajas de ahorro & 0,0070 & 0,0005 & 0,0061 & 0,0067 & 0,0070 & 0,0073 & 0,0079 \\
\hline $\begin{array}{l}\text { Cajas de ahorro } \\
\text { IRR }\end{array}$ & 1,0070 & 1,0061 & 1,0067 & 1,0070 & 1,0073 & 1,0079 & 1,0070 \\
\hline
\end{tabular}

FUENTE: Elaboración propia. 

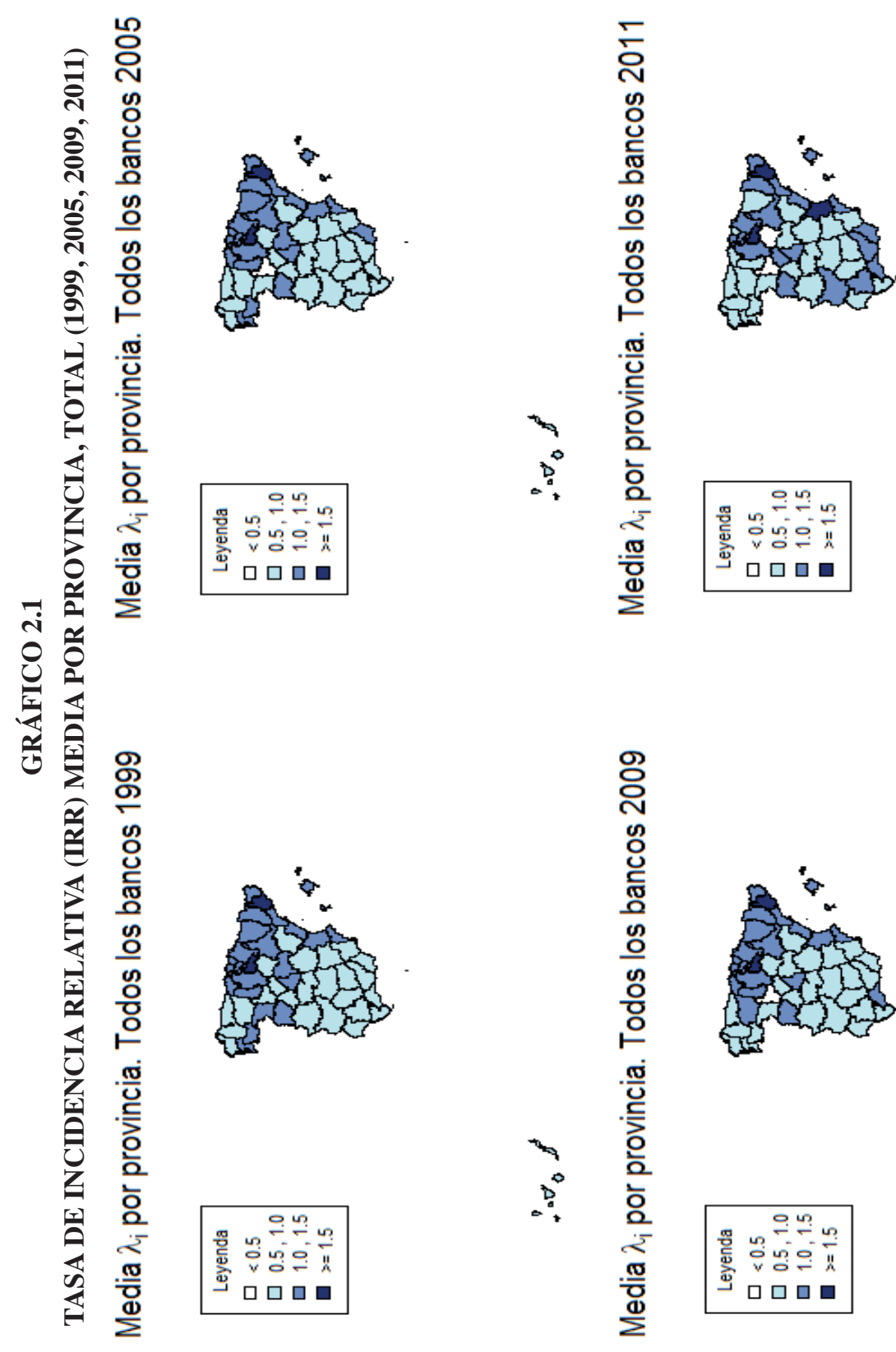

응

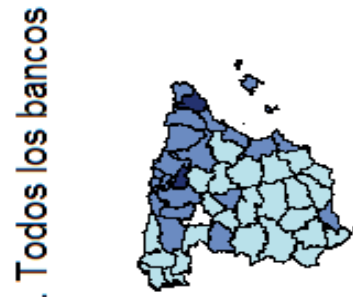

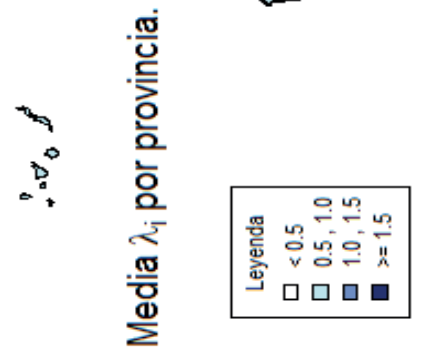



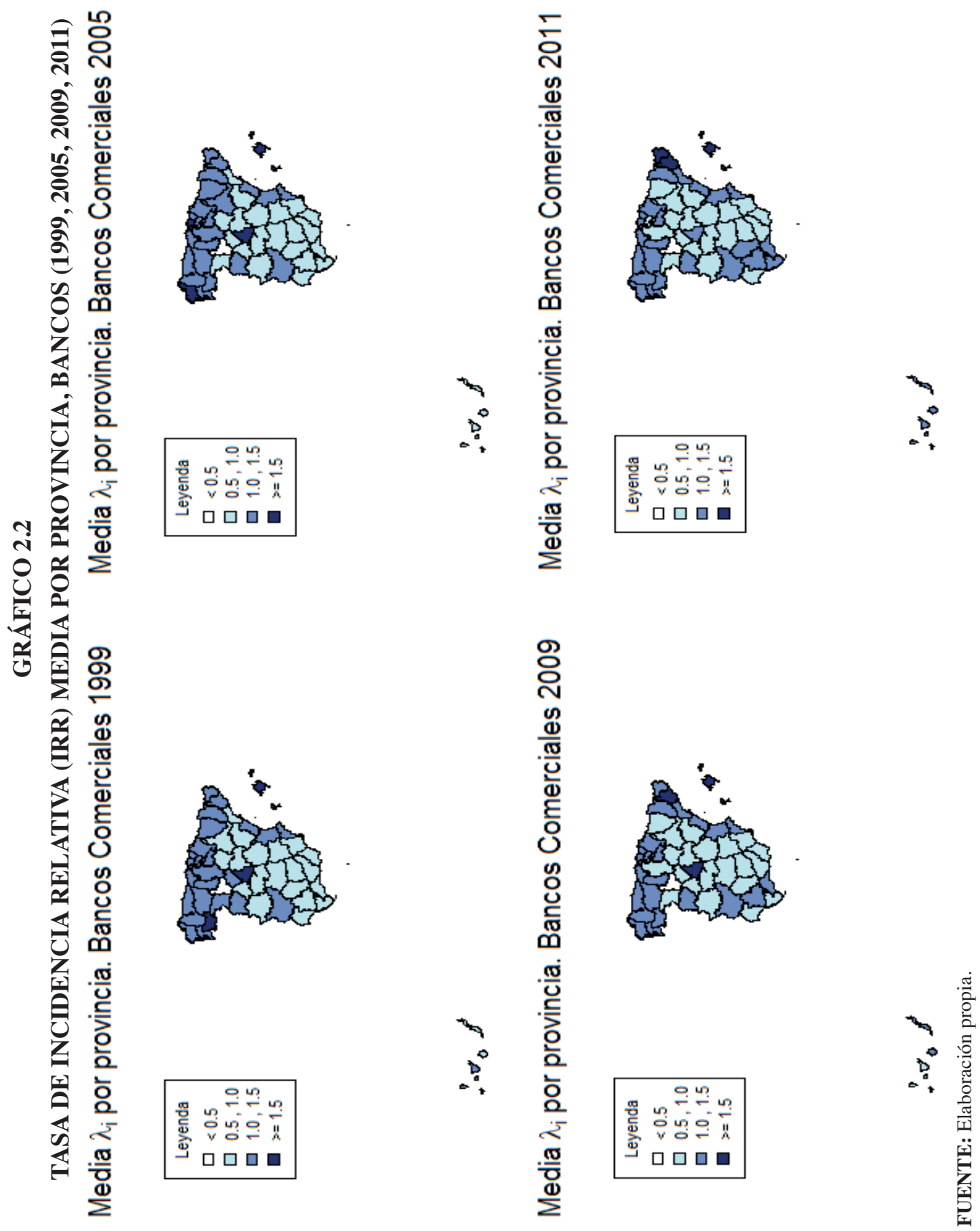


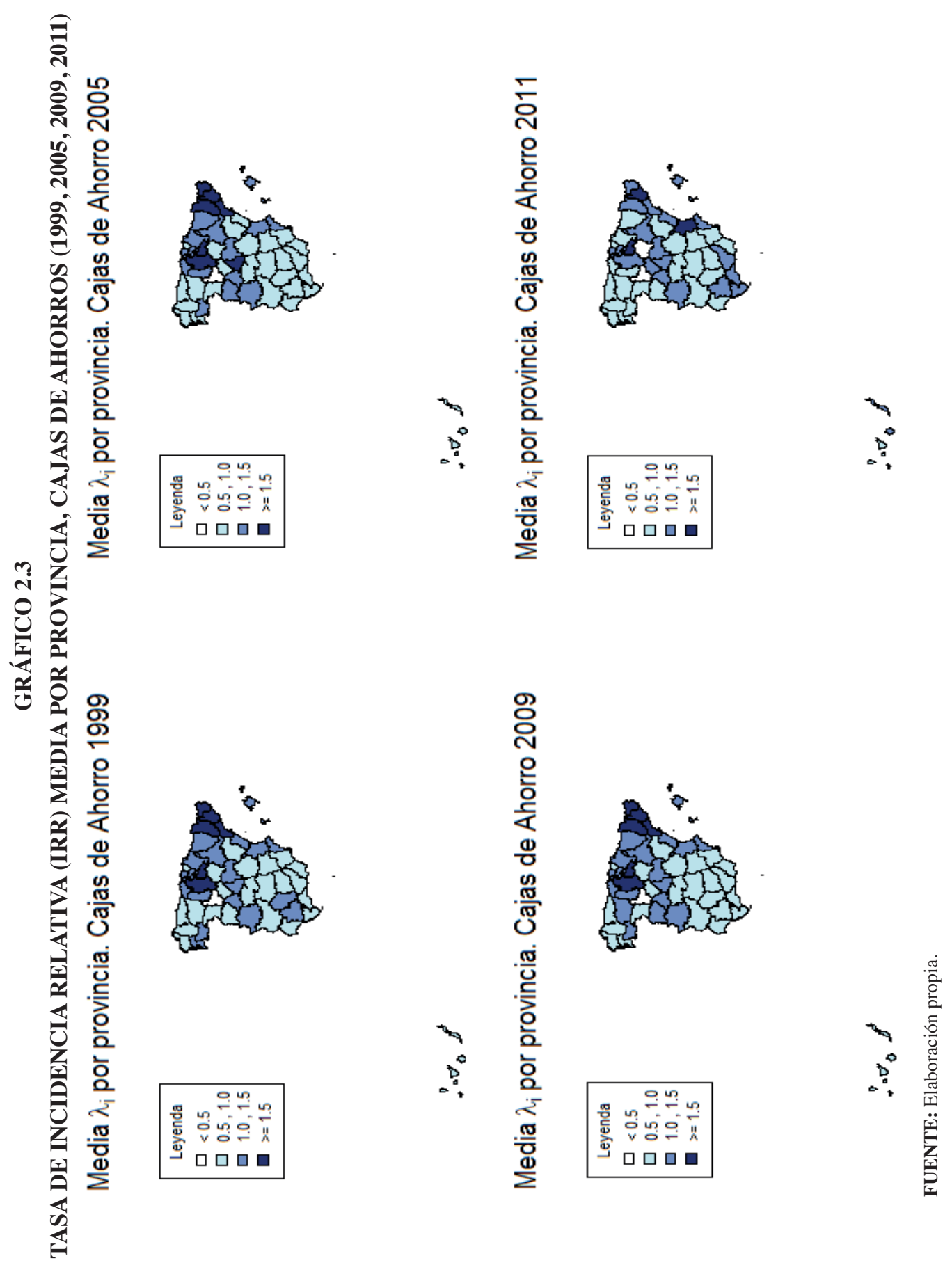




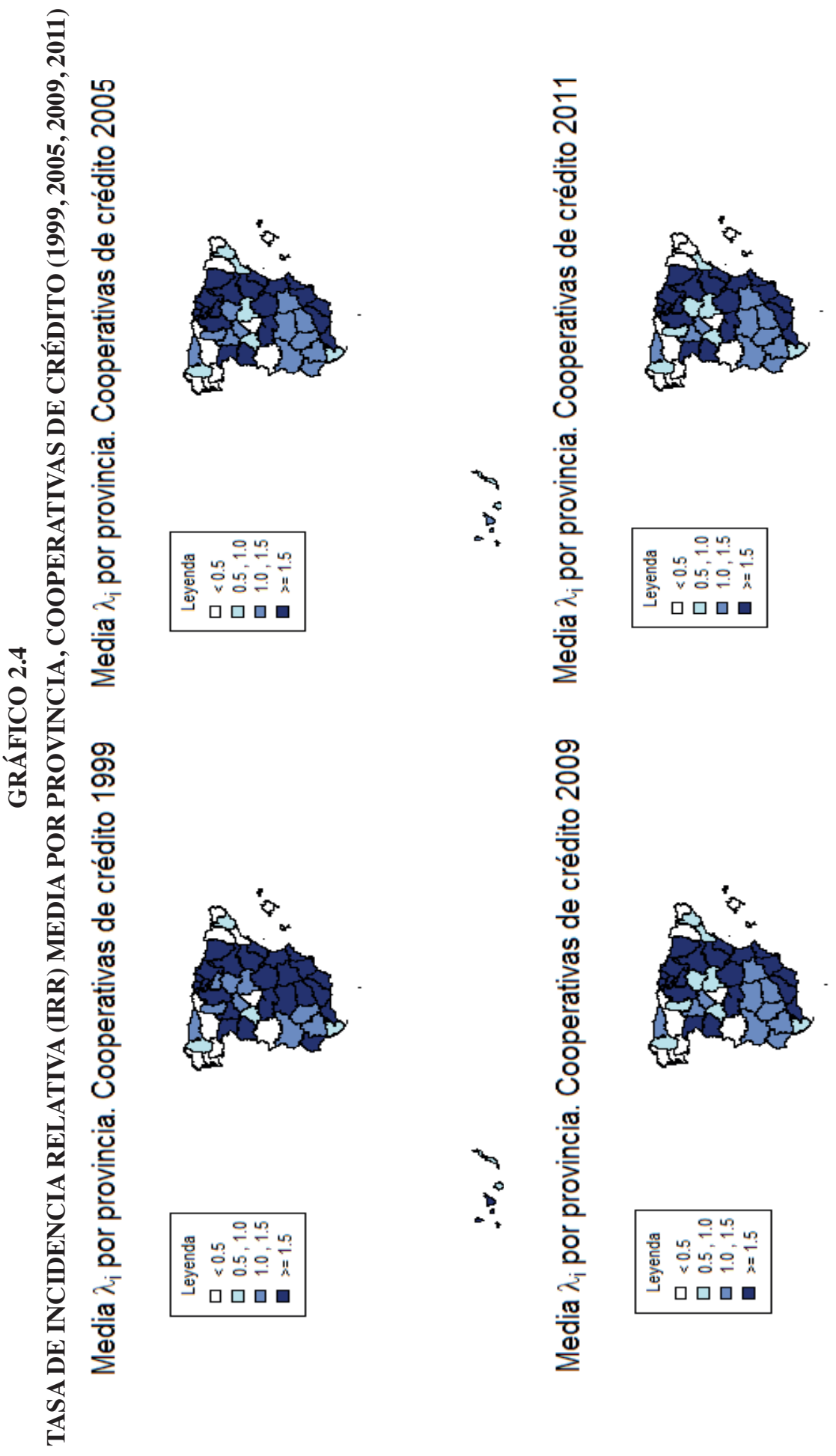




\section{Conclusiones}

Durante los últimos años, y tras el estallido de la crisis económico-financiera, los sistemas bancarios de gran parte de la Unión Europea se han visto seriamente afectados, desde numerosos puntos de vista. En el caso del sistema bancario español, el impacto ha sido de tal magnitud que, después de las intensas iniciativas desreguladoras de los años ochenta y noventa del siglo pasado, los efectos de la crisis sobre las entidades bancarias dieron lugar a una reestructuración y re-regulación del sector como ha sido la nueva Ley de Cajas (Ley 26/2013, de 27 de diciembre, de cajas de ahorros y fundaciones bancarias).

Dado lo reciente del proceso, que en determinados aspectos sigue sin haberse cerrado, nuevos análisis del sector que incluyan los años más recientes no son del todo viables por el momento, si bien existen ya numerosas contribuciones que han evaluado problemáticas concretas (véase, por ejemplo, y en relación con el presente trabajo (Maudos, 2017), que evalúa el fenómeno de la exclusión financiera hasta 2015). Entre los aspectos en los que el sector ha experimentado cambios más intensos y que, de hecho, todavía no han finalizado, se encuentra la expansión (hasta 2008) y posterior contracción en las redes de oficinas de bancos, cajas y cooperativas de crédito, especialmente estas dos últimas. Mientras que la desregulación de finales de los años ochenta del siglo pasado permitió a las cajas expandirse por todo el territorio nacional, emprendiendo políticas expansivas que se han definido incluso como agresivas (Illueca et al., 2009), desde el inicio de la crisis la tendencia ha sido exactamente la opuesta, si bien no ha habido una perfecta simetría (esto es, las posiciones relativas de las regiones donde más creció y disminuyó el número de oficinas no se han mantenido).

Ante el reto de la digitalización de los servicios bancarios, no es esperable que el proceso de racionalización en la red de oficinas bancarias vaya a detenerse en breve y, por tanto, resulta pertinente evaluar desde un punto de vista dinámico cuáles están siendo los patrones de localización de oficinas bancarias en España. Este ha sido el objeto de este trabajo en el que hemos modelizado el número de oficinas bancarias que podríamos esperar (en términos de población, y características socio-economicas) en una determinada localización geográfica, comparándolo con el de oficinas observadas para así cuantificar la posible existencia de exceso o falta de puntos de acceso físico a los servicios bancarios (definido en Alamá et al., 2015, como over-branching y under-branching).

Los resultados pueden ser valorados desde varios puntos de vista. Si bien el modelo utilizado tiene ciertas limitaciones en cuanto al número de covariables incluidas, no es posible una modelización más extensa por la escasez de información disponible a nivel municipal. Las tres variables consideradas (densidad de población, tasa de desempleo y porcentaje de población extranjera, así como el efecto del resto de entidades bancarias) tienen un impacto, en general, relevante, como muestran los intervalos de credibilidad (cuantiles) en todas las tablas de resultados. Sin embargo, existen diferencias notables cuando analizamos por separado los tres agregados 
institucionales, de manera que el exceso o carencia de oficinas (over-branching y under-branching) no sería un fenómeno achacable a los tres tipos de entidades por igual, siendo los bancos las entidades más implicadas en regiones con una mayor actividad económica. El factor temporal juega también un papel relevante y, en general, decreciente en el tiempo. Por último, el efecto aleatorio por provincia es el que nos indica si el número observado de oficinas es mayor o menor que el esperado según los niveles de población y las condiciones socio-económicas (representadas por las covariables) y es, por tanto, el que nos permite hablar de falta o exceso de oficinas (under- u over-branching).

Debido a la no disponibilidad de datos para algunas de las variables incluidas en el modelo, no ha sido posible extender el análisis hasta periodos más recientes, por lo que es inviable que las conclusiones puedan ir más allá del año 2011. Por tanto, ante la importancia que sigue teniendo el acceso físico a los servicios bancarios desde numerosos puntos de vista, y el hecho de que los procesos de contracción territorial de las redes de oficinas no parecen haber llegado a su fin, consideramos relevante la extensión de los análisis de los procesos localización bancaria, tanto para incluir periodos más recientes como para evaluar las implicaciones para la economía real.

\section{Referencias bibliográficas}

[1] ALAMÁ, L.; CONESA, D.; FORTE, A. y TORTOSA-AUSINA, E. (2015). «The geography of Spanish bank branches». Journal of Applied Statistics, 42 (4), 722-744.

[2] ALAMÁ, L. y TORTOSA-AUSINA, E. (2012). «Financial exclusion and bank branch geographic location patterns in Spain: 1988-2008». Growth and Change, 43 (3), 505543.

[3] BERNAD, C.; FUENTELSAZ, L. y GÓMEZ, J. (2008). «Deregulation and its longrun effects on the availability of banking services in low-income communities». Environment and Planning A, 40 (7), 1681-1696.

[4] BOOT, A. W. A. (2000). «Relationship banking: What do we know?». Journal of Financial Intermediation, 9, 7-25.

[5] BOOT, A. W. A. y THAKOR, A. V. (2000). «Can relationship banking survive competition?». The Journal of Finance, 55 (2), 679-713.

[6] CARBÓ, S.; GARDENER, E. P. M. y MOLYNEUX, P. (2005). Financial Exclusion. Palgrave MacMillan.

[7] CARBÓ, S.; GARDENER, E. P. M. y MOLYNEUX, P. (2007). «Financial exclusion in Europe». Public Money \& Management, 27 (1), 21-27.

[8] CHANG, A.; CHAUDHURI, S. y JAYARATNE, J. (1997). «Rational herding and the spatial clustering of bank branches: an empirical analysis». Research Paper 9724, Federal Reserve Bank of New York, New York, NY.

[9] DE JUAN, R. (2003). «The independent submarkets model: an application to the Spanish retail banking market». International Journal of Industrial Organization, 21, 1461-1487.

[10] DE JUAN, R. (2008). «Competition in local markets: Some evidence from the Spanish retail banking market». Review of Industrial Organization, 32 (2), 145-162. 
[11] DEGRYSE, H. y ONGENA, S. (2005). «Distance, lending relationships, and competition». The Journal of Finance, 60 (1), 231-266.

[12] DEMIRGÜÇ-KUNT, A. y LEVINE, R. (2001). Financial Structure and Economic Growth: A Cross Country Comparison of Banks, Markets and Development. MIT Press, Cambridge and London.

[13] ELYASIANI, E. y GOLDBERG, L. G. (2004). «Relationship lending: a survey of the literature». Journal of Economics and Business, 56, 315-330.

[14] ERGUNGOR, O. E. (2010). «Bank branch presence and access to credit in low-to moderateincome neighborhoods». Journal of Money, Credit and Banking, 42 (7), 13211349.

[15] FERNÁNDEZ-VILLAVERDE, J.; GARICANO, L. y SANTOS, T. (2013). «Political credit cycles: the case of the Eurozone». The Journal of Economic Perspectives, 27 (3), 145-166.

[16] FUENTELSAZ, L. y GÓMEZ, J. (2001). «Strategic and queue effects on entry in Spanish banking». Journal of Economics and Management Strategy, 10 (4), 529-563.

[17] FUENTELSAZ, L. y GÓMEZ, J. (2006). «Multipoint competition, strategic similarity and entry into geographic markets». Strategic Management Journal, 27, 477-499.

[18] FUENTELSAZ, L.; GÓMEZ, J. y POLO, Y. (2002). «Followers’ entry timing: Evidence from the Spanish banking sector after deregulation». Strategic Management Journal, 23 (3), 245-264.

[19] FUENTELSAZ, L.; GÓMEZ, J. y POLO, Y. (2004). «La expansión de la red de oficinas de las cajas de ahorros». Perspectivas del Sistema Financiero, 80, 57-71.

[20] GILJE, E. P.; LOUTSKINA, E. y STRAHAN, P. E. (2016). «Exporting liquidity: Branch banking and financial integration». The Journal of Finance, 71 (3), 1159-1183.

[21] GRÍA DE LA BANCA(VV.AA.). Cooperativas de Crédito y Cajas de Ahorros, Editorial Maestre Edibán, Madrid.

[22] HANNAN, T. H. y MCDOWELL, J. M. (1987). «Rival precedence and the dynamics of technology adoption: an empirical analysis». Economica, 54, 155-171.

[23] ILLUECA, M.; NORDEN, L. y UDELL, G. F. (2014). «Liberalization and risktaking: Evidence from government-controlled banks». Review of Finance, 18 (4), 1217 1257.

[24] ILLUECA, M.; PASTOR, J. M. y TORTOSA-AUSINA, E. (2009). «The effects of geographic expansion on the productivity of Spanish savings banks». Journal of Productivity Analysis, 32 (2), 119-143.

[25] JAIN, P. S. (1996). «Managing credit for the rural poor: lessons from the Grameen Bank». World Development, 24 (1), 79-89.

[26] JAYARATNE, J. y STRAHAN, P. E. (1996). «The finance-growth nexus: Evidence from bank branch deregulation». The Quarterly Journal of Economics, 111 (3), 639670.

[27] JOASSART-MARCELLI, P. y STEPHENS, P. (2010). «Immigrant banking and financial exclusion in Greater Boston». Journal of Economic Geography, 10 (6), 883-912.

[28] KING, R. G. y LEVINE, R. (1993). «Finance and growth: Schumpeter might be right». The Quarterly Journal of Economics, 108 (3), 717-737.

[29] LANZILLOTTI, R. F. y SAVING, T. R. (1969). «State branching restrictions and the availability of branching services: Comment». Journal of Money, Credit and Banking, 1 (4), 778-788. 
[30] LEYSHON, A. y THRIFT, N. (1995). «Geographies of financial exclusion: financial abandonment in Britain and the United States». Transactions of the Institute of British Geographers, New Series, 20, 312-341.

[31] LEYSHON, A. y THRIFT, N. (1996). «Financial exclusion and the shifting boundaries of the financial system». Environment and Planning A, 28 (7), 1150-1156.

[32] LOUTSKINA, E. y STRAHAN, P. E. (2015). «Financial integration, housing and economic volatility». Journal of Financial Economics, 115 (1), 25-41.

[33] MAUDOS, J. (2017). «Bank restructuring and access to financial services: the Spanish case». Growth and Change (en prensa).

[34] McCULLOCH, R. E. y SEARLE, S. R. (2001). Generalized, Linear and Mixed Models, John Wiley and Sons.

[35] MORDUCH, J. (1999). «The microfinance promise». Journal of Economic Literature, 37 (4), 1569-1614.

[36] OKEAHALAM, C. (2009). «Bank branch location: a count analysis». Spatial Economic Analysis, 4 (3), 275-300.

[37] ONGENA, S. y SMITH, D. C. (2000). «Bank relationships: a review», en P. T. Harker y S. A. Zenios (eds.), Performance of Financial Institutions. Efficiency, Innovation, Regulation, cap. 7, pp. 221-258. Cambridge University Press, Cambridge.

[38] ONGENA, S. y SMITH, D. C. (2001). «The duration of bank relationships». Journal of Financial Economics, 61, 449-475.

[39] PASTOR, J. M.; PAVÍA, J. M.; SERRANO, L. y TORTOSA-AUSINA, E. (2017). «Rich regions, poor regions, and bank branch deregulation in Spain». Regional Studies (en prensa).

[40] PETERSEN, M. A. y RAJAN, R. G. (1994). «The benefits of lending relationships: evidence from small business data». Journal of Finance, 49 (1), 3-37.

[42] PETERSEN, M. A. y RAJAN, R. G. (1995). «The effect of credit market competition on lending relationships». Quarterly Journal of Economics, 110 (2), 407-443.

[43] POLLARD, J. S. (1996). «Banking at the margins: a geography of financial exclusion in Los Angeles». Environment and Planning A, 28, 1209-1232.

[44] RUE, H.; MARTINO, S. y CHOPIN, N. (2009). «Approximate Bayesian inference for latent Gaussian models by using integrated nested Laplace approximations». Journal of the Royal Statistical Society: Series B (Statistical Methodology), 71 (2), 319-392.

[45] SUTTON, J. (1997). «One smart agent». Rand Journal of Economics, 28 (4), 605-628. 